\title{
A systematic review of correlates of sedentary behaviour in adults aged 18-65 years: a socio-ecological approach
}

Grainne O'Donoghue ${ }^{1 * \dagger}$, Camille Perchoux ${ }^{2}$, Keitly Mensah2 ${ }^{2}$, Jeroen Lakerveld ${ }^{3}$, Hidde van der Ploeg ${ }^{3}$, Claire Bernaards ${ }^{4}$, Sebastien F. M. Chastin ${ }^{5}$, Chantal Simon², Donal O'Gorman', Julie-Anne Nazare ${ }^{2 \dagger}$, on behalf of the DEDIPAC consortium

\begin{abstract}
Background: Recent research shows that sedentary behaviour is associated with adverse cardio-metabolic consequences even among those considered sufficiently physically active. In order to successfully develop interventions to address this unhealthy behaviour, factors that influence sedentariness need to be identified and fully understood. The aim of this review is to identify individual, social, environmental, and policy-related determinants or correlates of sedentary behaviours among adults aged 18-65 years.

Methods: PubMed, Embase, CINAHL, PsycINFO and Web of Science were searched for articles published between January 2000 and September 2015. The search strategy was based on four key elements and their synonyms: (a) sedentary behaviour (b) correlates (c) types of sedentary behaviours (d) types of correlates. Articles were included if information relating to sedentary behaviour in adults (18-65 years) was reported. Studies on samples selected by disease were excluded. The full protocol is available from PROSPERO (PROSPERO 2014:CRD42014009823).

Results: 74 original studies were identified out of 4041: 71 observational, two qualitative and one experimental study. Sedentary behaviour was primarily measured as self-reported screen leisure time and total sitting time. In 15 studies, objectively measured total sedentary time was reported: accelerometry $(n=14)$ and heart rate $(n=1)$. Individual level factors such as age, physical activity levels, body mass index, socio-economic status and mood were all significantly correlated with sedentariness. A trend towards increased amounts of leisure screen time was identified in those married or cohabiting while having children resulted in less total sitting time. Several environmental correlates were identified including proximity of green space, neighbourhood walkability and safety and weather.

Conclusions: Results provide further evidence relating to several already recognised individual level factors and preliminary evidence relating to social and environmental factors that should be further investigated. Most studies relied upon cross-sectional design limiting causal inference and the heterogeneity of the sedentary measures prevented direct comparison of findings. Future research necessitates longitudinal study designs, exploration of policy-related factors, further exploration of environmental factors, analysis of inter-relationships between identified factors and better classification of sedentary behaviour domains.
\end{abstract}

Keywords: Sitting, Sedentary behaviour, Determinants, Correlates, Adults, Ecological model, Intrapersonal, Interpersonal, Environment, Policy-related

\footnotetext{
* Correspondence: grainne.odonoghue@dcu.ie

${ }^{\dagger}$ Equal contributors

${ }^{1}$ Centre for Preventive Medicine, School of Health \& Human Performance,

Dublin City University, Dublin 9, Republic of Ireland

Full list of author information is available at the end of the article
} 


\section{Background}

The time that adults spend sedentary or put simply doing "too much sitting" has recently been proposed as a population wide issue that has deleterious effects on health outcomes. New evidence links excessive sitting in adults with lifestyle related diseases such as obesity, type II diabetes, cardiovascular diseases, pulmonary disease and cancer [1-3]. It has been shown that sedentary time has specific metabolic consequences even among those meeting the moderate-to-vigorous physical activity guidelines. A gradient exists with higher morbidity and mortality rates among those who spend more of their time being sedentary, independent of whether or not they engage in regular physical activity [2, 3]. Typically, "sedentary" is defined as any waking activity that requires an energy expenditure ranging from 1.0 to 1.5 (basal metabolic rate) while in a sitting or reclining posture $[4,5]$.

The focus to date on factors that influence sedentary behaviours has mostly been on individual level factors such as biological, psychological and behavioural $[1,6]$. However, it has become apparent that these are not stand-alone factors and addressing them in isolation will not result in a significant change in sedentary behaviours [1]. Social, environmental and policy factors may also need to be taken into account. The current rationale is that factors that influence sedentary behaviour can be conceptualised using models such as the socio-ecological model [6]. The socio-ecological approach emphasises that focus should not only be on individual behavioural factors but also on the multiple-level factors that influence the specific behaviour in question [7], thus focusing on the interrelationships between individuals and the social, physical and policy environment. This model places the individual within an ecosystem that acknowledges individual behaviour is dependent on the dynamic relationships between it and other determinants or correlates relating to the environment, economy, political and social agendas [7]. The model has been widely applied to research looking at what influences physical activity behaviours [7] and it has been suggested that a comprehensive approach, such as that offered by the socioecological model is essential for examining the multiple level factors that might determine sedentary behaviours [1]. This ecological model provides a framework that facilitates mapping the multiple domains of sedentary behaviour, while at the same time assuming multiple levels of influence [1].

A previous review investigating sedentary behaviour correlates in adults identified numerous intrapersonal factors relating to sedentary behaviour, several which are non-modifiable (for example, age and gender) [6]. However they did not identify many factors or correlates outside of the individual. Potentially significant factors such as the built, physical, social and policy environments need to be identified and since the publication of that review there have been several studies investigating the environmental influences on sedentary behaviours, both at an individual and community level [8-18]. These factors need systematic identification so that they can be considered along with individual level and social correlates in the development of interventions to address sedentary behaviours. Therefore, the aim of this study is to comprehensively review the quantitative (observational and experimental) and qualitative literature on determinants and correlates of sedentary behaviours in adults aged between 18 and 65 years. The overall objectives are to (i) provide an update on previously reported factors, (ii) identify novel intrapersonal (individual), interpersonal (social), environment and policy factors, (iii) investigate the interactions between the different factors, (iv) identify gaps in the existing literature and (v) provide recommendations for future research in this area.

\section{Methods}

This systematic review is one of three reviews, part of the Joint Programming Initiative's funded Determinants of Diet and Physical Activity (DEDIPAC) consortium [19] aimed at reviewing and updating the current evidence base on the determinants and correlates of sedentary behaviour across the life course, with two other reviews focusing on children and adolescents ( $<18$ years) and older adults ( $>65$ years). A common protocol for the three DEDIPAC systematic reviews across the life course was developed and is available from PROSPERO (PROSPERO 2014:CRD42014009823).

\section{Search strategy}

Five electronic databases (PubMed, Embase, CINAHL with full text, PsycINFO and Web of Science) were searched. The search strategy was based on four key elements: (a) sedentary behaviour and its synonyms (e.g., sedentariness); (b) correlates or determinants and its synonyms (e.g., correlates, factors); (c) types of sedentary behaviour (e.g., TV viewing, gaming) and (d) possible correlates or determinants of sedentary behaviour (e.g., environmental, behavioural and socio-demographic). Terms referring to these four elements were used as MESH-headings and title or abstract words in all databases. A complete list of the search terms is available in the additional materials section (Additional file 1: Table S1). In addition to the above, the reference lists of all included articles were scanned for articles that met the inclusion criteria. Any retrieved articles underwent the same selection process as the other articles.

\section{Inclusion criteria}

Scientific peer reviewed published papers written in English from January 2000-September 2015 were included in the review (conference abstracts, reports and thesis were 
excluded). Adults were defined as any population aged $\geq 18$ years and $<65$ years. Articles whose primary outcome focuses on specific patient groups/pathology were excluded. Study designs eligible for inclusion were observational studies (cross sectional, case control and prospective), experimental studies (randomised controlled trials, quasi-experimental trials) and qualitative studies. In terms of sedentary behaviour outcome measures, one or more of the following were acceptable; total sedentary or sitting time (e.g., minutes per day) or time spent in one or more of the following specific domains of sedentary behaviour; time spent watching TV, screen time (in any domain i.e., leisure or work), occupational sitting time or transport related sitting time. Both objective and subjective measurement outcomes were included (cut off point for accelerometric sedentary behaviour $=\leq 100$ counts per minute).

\section{Selection process}

The selection process consisted of three phases. In the initial phase, two reviewers (GO'D and KM) independently screened the yielded articles based on title. In the case of doubt, the articles were included in the abstract review phase. In phase two, all articles selected from the initial phase had their abstract reviewed and assessed by three independent reviewers (GO'D, JAN and CP). Any disagreement was resolved by the third reviewers (JL, $\mathrm{HvdP}$ and $\mathrm{CB}$ ). In the final phase, the remaining articles were fully reviewed by the same three independent reviewers using the pre-determined inclusion criteria. Any disagreement between reviewers in this phase was resolved by discussion within the wider team.

\section{Data extraction}

An eight item standardised pre-piloted data extraction form was used to extract data from the included studies under the following headings: (i) general information; (ii) sample characteristics; (iii) study design; (iv) measurement of sedentary behaviour; (v) measures of factors that influence sedentary behaviour; (vi) statistical analyses; (vii) results reported and (viii) general conclusions. Additional file 1: Table S2 (additional files) provides further detail.

\section{Risk of bias}

To assess the risk of bias, the quality assessment tool 'QUALSYST' from the "Standard Quality Assessment Criteria for Evaluating Primary Research Papers from a Variety of Fields" (Alberta Heritage Foundation for Medical Research) was used. This pragmatic tool incorporates two scoring systems, allowing quality assessment to be conducted on both quantitative and qualitative research [20]. As both quantitative and qualitative study designs were included in this review, this tool was deemed appropriate (Additional file 1: Tables S3 and S4). Fourteen items for each quantitative study and 10 for each qualitative study were scored depending on the degree to which the specific criteria were met or reported ('yes' $=2$, 'partial' $=1$, 'no' $=0$ ). Items not applicable to a particular study design were marked ' $\mathrm{n} / \mathrm{a}$ ' and excluded when calculating the summary score. The three reviewers involved in article selection assessed quality independently (GO'D, JAN and CP). All articles were reviewed by at least two of the three reviewers. A quality assurance process enabled cross checking of quality assessment. Discrepancies were resolved through discussion.

\section{Data synthesis}

A narrative synthesis of the findings of the review is provided structured around the ecological model of sedentary behaviour [1]. A narrative synthesis was conducted because of the high levels of clinical, methodological and statistical heterogeneity, making data pooling inappropriate. Qualitative tables illustrate the main study characteristics and show the individual, social and environmental factors that have been investigated and their relationships to sedentary behaviour. Direction and strength of the association between these factors and the different categories of sedentary behaviour are summarised, as well as the gender categories under study. A thematic synthesis was used to summarise the qualitative studies and the findings are integrated with the quantitative findings using the parallel synthesis approach recommended for mixedmethods research synthesis [21].

\section{Results}

The process for undergoing the literature search and screening, including numbers of papers excluded and reasons for exclusion is illustrated in Fig. 1. In summary, the electronic search yielded 4584 records and a manual search of personal databases and recent publication reference lists yielded a further 40 records, resulting in a total of 4624 records. 583 duplicates were removed. Of the remaining 4041 records, 3967 were excluded throughout the screening process. Overall, 74 papers passed the eligibility criteria to be included in the review.

\section{Study characteristics}

Table 1 provides a detailed overview of all the included study characteristics. Of the 74 studies included, 21 were conducted in North America, 23 in Europe, 24 in Australia, one in New Zealand and one across three continents (United States, Australia and Belgium). The remaining four were conducted in Asia. All studies apart from three (one experimental and two qualitative) were observational. The most common observational study design identified was cross sectional $(n=58)$. Participant sample sizes ranged from 10 to 246,920 adults with age ranging from 18 to 


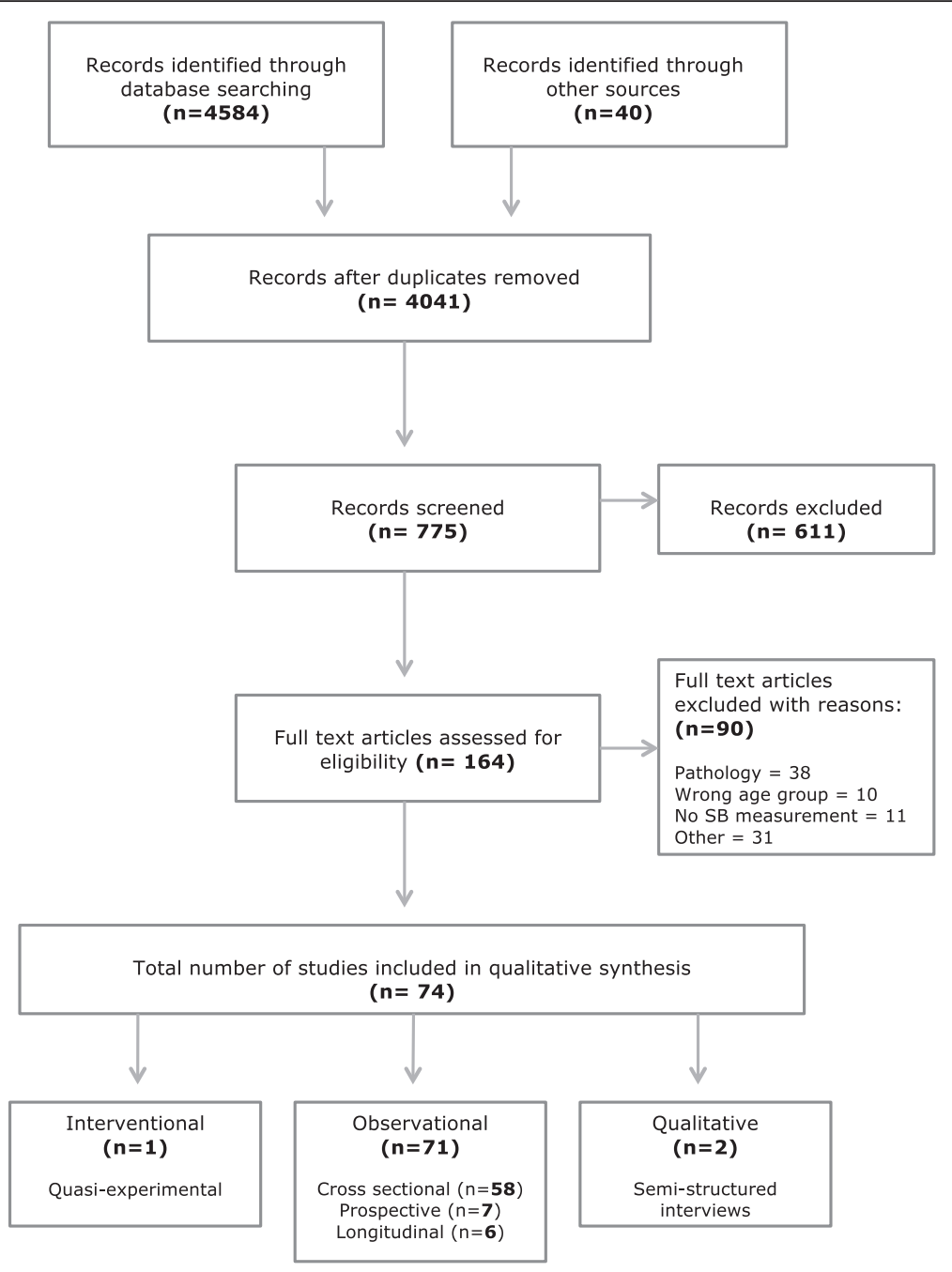

Fig. 1 PRISMA diagram of study selection process

65 years in all studies but one where the age ranged from 16 to 96 years [22]. In terms of gender, eleven studies were based on women only and two on men while the remainder included both. Participants from a broad range of socioeconomic backgrounds were included across the various studies with only eight addressing specific working groups.

\section{Risk of bias}

The quality scores for the included 74 studies, expressed as a percentage (with $0 \%$ the worst and $100 \%$ the best possible quality), ranged from 41 to $95 \%$ as illustrated in Table 1. Overall the studies were of good quality with a median score of $85 \%$. Of all the items on the checklist for the quantitative study quality assessment, items 1 'question/objective sufficiently described', item 2 'study design evident and appropriate' and item 10 'analytic methods described/justified and appropriate' were the most frequently reported. Item 11 'some estimate of variance is reported for the main results' appeared to be the item most frequently missing.

\section{Measurement of sedentary behaviours}

In total, 16 studies objectively measured sedentary time with fifteen using accelerometry (ActiGraph $n=14$ and activPAL $n=1$ ) and one using heart rate. Seven studies used both self-report and objective measures, and the remainder relied upon self-reported sedentary time measurement $(n=58)$. Five domains of self-report sedentariness have been identified (some studies report more than one domain):

1. Total screen time

2. Television and screen entertainment (TVSE)

3. Transport sitting time

4. Total sitting time (including occupational sitting)

5. Leisure sitting time (time outside of work, TVSE, reading/listening to music/socialising) 
Table 1 Overview of study characteristics

\begin{tabular}{|c|c|c|c|c|c|c|c|}
\hline Author ${ }^{\mathrm{REF}}$ & $\begin{array}{l}\text { Number, age, } \\
\text { gender }\end{array}$ & Design & Outcome & Individual factors & Environmental factors & Interpersonal factors & $\begin{array}{l}\text { Quality } \\
\text { score }\end{array}$ \\
\hline Astell-Burt [14] & $\begin{array}{l}246920 \text { adults } \\
74-106 \text { years } \\
48 \% \text { men }\end{array}$ & CS & Sitting time & & $\begin{array}{l}\text { Proximity of green } \\
\text { spaces }\end{array}$ & & 0.86 \\
\hline Ballard [35] & $\begin{array}{l}116 \text { men } \\
\text { Mean } \\
\text { age }=19.54\end{array}$ & CS & $\begin{array}{l}\text { TV Viewing } \\
\text { Video games } \\
\text { Reading }\end{array}$ & $\begin{array}{l}\text { BMI, body fat } \% \text {, frequency of } \\
\text { exercise, length of exercise, } \\
\text { days of moderate activity, } \\
\text { days of walking }\end{array}$ & & & 0.86 \\
\hline Barnett [73] & $\begin{array}{l}3334 \text { adults } \\
45-79 \text { years } \\
48 \% \text { men }\end{array}$ & $\mathrm{PO}$ & Changes in TV viewing time & $\begin{array}{l}\text { Age, retirement, social class, } \\
\text { levels of PA }\end{array}$ & & & 0.90 \\
\hline Bowman [32] & $\begin{array}{l}9157 \\
\geq 20 \text { years }\end{array}$ & CS & TV Viewing & $\begin{array}{l}\text { Age, sex, education, race, } \\
\text { ethnicity }\end{array}$ & & & 0.86 \\
\hline Chau [38] & $\begin{array}{l}10785 \text { adults } \\
15-69 \text { years } \\
42 \% \text { men }\end{array}$ & CS & $\begin{array}{l}\text { Leisure sitting time } \\
\text { Sitting time at work }\end{array}$ & Occupational activity & & & 0.90 \\
\hline Clark [68] & $\begin{array}{l}\text { Young cohort: } \\
n=5215 \text {, age } \\
24.6(1.5) \\
\text { mid-aged cohort: } \\
n=6973 \text {, age } \\
52.5(1.4) \\
100 \% \text { women }\end{array}$ & P & $\begin{array}{l}\text { Hours per day total sitting } \\
\text { (visiting friends, reading, } \\
\text { driving, reading, watching } \\
\text { TV or working at desk/computer) } \\
\text { on week and weekend days }\end{array}$ & $\begin{array}{l}\text { Life events in the previous } \\
12 \text { months: major illness } \\
\text { surgery, return to study, } \\
\text { moving out, decreased } \\
\text { income, menopause, }\end{array}$ & & $\begin{array}{l}\text { Life events in the previous } \\
12 \text { months: decline health } \\
\text { of close family, birth of child, } \\
\text { begin work, loss of job, change } \\
\text { at work, divorce, new relationship, } \\
\text { retirement, spouse retirement, } \\
\text { child leaving home }\end{array}$ & 0.84 \\
\hline Clark [48] & $\begin{array}{l}10951 \text { adults } \\
25-91 \text { years } \\
45 \% \text { men }\end{array}$ & CS & Time spent in TVSE & $\begin{array}{l}\text { Age, education, household } \\
\text { income, employment status }\end{array}$ & $\begin{array}{l}\text { Living outside the state } \\
\text { capital city }\end{array}$ & Living arrangements & 0.95 \\
\hline Clemes [39] & $\begin{array}{l}170 \text { adults } \\
18-65 \text { years } \\
30 \% \text { men }\end{array}$ & CS & Time spent sedentary ${ }^{\circ}$ & Levels of PA outside work & & Workdays vs. non-workdays & 0.77 \\
\hline Coogan [8] & $\begin{array}{l}59000 \text { women } \\
21-69 \text { years }\end{array}$ & CS & TV Viewing & & $\begin{array}{l}\text { Neighbourhood walkability, } \\
\text { neighbourhood SES }\end{array}$ & & 0.81 \\
\hline Conroy [45] & $\begin{array}{l}128 \text { adults } \\
\text { Mean age }=31,3 \\
(\mathrm{SD}=1,1) \\
41 \% \text { men }\end{array}$ & CS & Time spent sedentary ${ }^{\circ}$ & $\begin{array}{l}\text { Sedentary habits, daily } \\
\text { intentions to limit sedentary } \\
\text { behaviour, levels of PA }\end{array}$ & & & 0.86 \\
\hline Crespo $[86]^{\mathrm{a}}$ & $\begin{array}{l}1313 \text { adults } \\
\text { Mean age = } 45 \\
(\mathrm{SD}=10) \\
56 \% \text { men }\end{array}$ & CS & Time spent sedentary ${ }^{\circ}$ & $\begin{array}{l}\text { Age, gender, education, } \\
\text { ethnicity }\end{array}$ & $\begin{array}{l}\text { Worksite promotion index } \\
\text { including: shower facilities } \\
\text { at work, lockers for clothes } \\
\text { at work, safe bicycle } \\
\text { storage }\end{array}$ & & 0.95 \\
\hline De Cocker [64] & 5562 women & L & Changes in sitting time & Weight & & & 0.91 \\
\hline
\end{tabular}


Table 1 Overview of study characteristics (Continued)

\begin{tabular}{|c|c|c|c|c|}
\hline De Cocker [54] & $\begin{array}{l}993 \text { adults mean } \\
\text { age } 51\end{array}$ & CS & Occupational sitting time & $\begin{array}{l}\text { Gender, age, educational level, } \\
\text { household income, self-reported } \\
\text { health, self-efficacy about sitting } \\
\text { less, intention to sit less }\end{array}$ \\
\hline De Wit [57] & $\begin{array}{l}3005 \text { adults } \\
18-65 \text { years } \\
34 \% \text { men }\end{array}$ & CS & $\begin{array}{l}\text { Time spent watching TV or } \\
\text { using PC }\end{array}$ & $\begin{array}{l}\text { Depressive symptoms, anxiety } \\
\text { disorders }\end{array}$ \\
\hline Den Hoed [69] & $\begin{array}{l}1654 \text { adults twins } \\
2 \% \text { men } \\
\text { Mean age }=56 \\
(\mathrm{SD}=10)\end{array}$ & CS & Time spent sedentary ${ }^{\circ}$ & $\begin{array}{l}\text { Heritability (additive genetic } \\
\text { factors) }\end{array}$ \\
\hline Ding [9] & $\begin{array}{l}551 \text { adults } \\
20-70 \text { years olds } \\
39 \% \text { men }\end{array}$ & $\mathrm{L}$ & Changes in TV viewing time & $\begin{array}{l}\text { Age, gender, education, annual } \\
\text { household income, employment } \\
\text { status, occupational PA, } \\
\text { domestic PA, transport PA }\end{array}$ \\
\hline Ding [26] & $\begin{array}{l}37570 \text { adults average } \\
\text { age } 61 \text { year, } 54 \% \\
\text { female }\end{array}$ & CS & $\begin{array}{l}\text { Time spent driving (motorised } \\
\text { transport) }\end{array}$ & $\begin{array}{l}\text { Smoking, alcohol consumption, } \\
\text { dietary risk, physical activity } \\
\text { levels, sleep quality, BMI, quality } \\
\text { of life, self-rated health }\end{array}$ \\
\hline Ekelund [56] & $\begin{array}{l}393 \text { adults } \\
\text { Mean age }=49,7 \\
(\mathrm{SD}=8) \\
45 \% \text { men }\end{array}$ & $P$ & Time spent sedentary ${ }^{\circ}$ & BMI, fat mass, waist circumference \\
\hline Evenson [70] & $\begin{array}{l}359 \text { women } \\
\geq 16 \text { years }\end{array}$ & CS & Time spent sedentary $^{\circ}$ & Pregnancy \\
\hline Fields [11] & $\begin{array}{l}189 \text { adults } \\
\text { Mean age = } 32 \\
(\mathrm{SD}=10,2) \\
31 \% \text { men }\end{array}$ & CS & $\begin{array}{l}\text { Time spent sedentary outside } \\
\text { of work }\end{array}$ & \\
\hline Frank [83] & $\begin{array}{l}10876 \text { adults } \\
46 \% \text { male }\end{array}$ & CS & Car time as passenger or driver & \\
\hline George [67] & $\begin{array}{l}15 \text { men } \\
35-64 \text { years }\end{array}$ & Q & $\begin{array}{l}\text { Barriers to decreasing sedentary } \\
\text { time }\end{array}$ & Health status and working hours \\
\hline Granner [31] & $\begin{array}{l}189 \text { women } \\
18-60 \text { years }\end{array}$ & CS & $\begin{array}{l}\text { TV viewing } \\
\text { Sitting time } \\
\text { Time spent sedentary }\end{array}$ & $\begin{array}{l}\text { Age, education, employment } \\
\text { status, ethnicity, eat meals or } \\
\text { snacks while watching TV, BMI, } \\
\text { self-rated health, number of days } \\
\text { per month depressed, number } \\
\text { of days per month anxious }\end{array}$ \\
\hline
\end{tabular}

Social norm towards sitting less in work, social support towards sitting less in work

Neighbourhood walkability Living arrangements, number index, neighbourhood of children ( $<18$ years) in the pedestrian infrastructures, householdSocial interactions aesthetics, traffic-related and social cohesion, sense of safety, crime-related safety- community safety

Land use mix, intersection, density, residential density

Weather as a barrier, access Social interactions and sense of to recreation facilities community and family support 
Table 1 Overview of study characteristics (Continued)

\begin{tabular}{|c|c|c|c|c|c|c|c|}
\hline Grothe [30] & $\begin{array}{l}39 \text { women } \\
\geq 18 \text { years }\end{array}$ & CS & $\begin{array}{l}\text { Time spent sedentary } \\
\text { TV ViewingVideo games } \\
\text { Computer work } \\
\text { Paper work } \\
\text { Phone use } \\
\text { Reading } \\
\text { Doing artwork } \\
\text { Transportation sitting time }\end{array}$ & $\begin{array}{l}\text { Age, education, income, ethnicity, } \\
\text { food cravings, BMI, illness }\end{array}$ & & & 0.90 \\
\hline Hadgraft [40] & $\begin{array}{l}1235 \text { adults mean } \\
\text { age } 53.738 \% \\
\text { women }\end{array}$ & CS & $\begin{array}{l}\text { Occupational sitting time and TV } \\
\text { viewing time }\end{array}$ & $\begin{array}{l}\text { Income, profession, energy intake, } \\
\text { educational attainment, leisure } \\
\text { time physical activity, BMl }\end{array}$ & & Marital status & 0.90 \\
\hline $\begin{array}{l}\text { Hagströmer } \\
{[13]}\end{array}$ & $\begin{array}{l}1172 \text { adults } \\
19-69 \text { years } \\
45 \% \text { men }\end{array}$ & CS & Time spent sedentary $^{\circ}$ & & Region, season & & 0.81 \\
\hline Hamer [29] & $\begin{array}{l}3923 \text { adults } \\
\text { Mean age }=51 \\
(\mathrm{SD}=15,8)\end{array}$ & CS & Time spent in TVSE & $\begin{array}{l}\text { Deprivation, BMI, mental health, } \\
\text { physical function, psychological } \\
\text { distress, smoking, alcohol intake, } \\
\text { fruits and vegetables intake }\end{array}$ & & & 0.86 \\
\hline Hamrik [50] & $19-90$ years & CS & Time spent sedentary & Age, gender & & & 0.7 \\
\hline Hirooka [43] & $\begin{array}{l}97 \text { adults } \\
\geq 18 \text { years } \\
41 \% \text { men }\end{array}$ & CS & $\begin{array}{l}\text { Sitting/lying timeTV/computer } \\
\text { time }\end{array}$ & $\begin{array}{l}\text { Total time in exercise, localization } \\
\text { (Japan vs. USA) }\end{array}$ & & & 0.8 \\
\hline Ishii [63] & $\begin{array}{l}1034 \text { adults } \\
40-69 \text { years } \\
52 \% \text { men }\end{array}$ & CS & Time spent in TVSE & $\begin{array}{l}\text { Age, gender, education, household } \\
\text { income, employment statusBMl }\end{array}$ & & $\begin{array}{l}\text { Living arrangements, marital } \\
\text { status }\end{array}$ & 0.90 \\
\hline Jans [74] & $\begin{array}{l}7720 \text { adults } \\
\text { Mean age }=32 \\
(S D=11) \\
60 \% \text { men }\end{array}$ & $\mathrm{CS}$ & $\begin{array}{l}\text { Total sedentary time } \\
\text { Total sitting time } \\
\text { Sitting time at work } \\
\text { Sitting time commuting } \\
\text { Sitting time during house work } \\
\text { Sitting time during the day/ } \\
\text { evening }\end{array}$ & $\begin{array}{l}\text { Occupational groups, business } \\
\text { sectors }\end{array}$ & & & 0.72 \\
\hline Kaufman [33] & $>20$ years & $\mathrm{CS}$ & $\begin{array}{l}\text { Time spent sedentary outside } \\
\text { of work }\end{array}$ & Smoking & & & 0.86 \\
\hline Kozo [59] & $\begin{array}{l}2196 \text { adults } \\
\text { Mean age }=45 \\
(\mathrm{SD}=11) \\
51 \% \text { years }\end{array}$ & CS & $\begin{array}{l}\text { Time spent sedentary } \\
\text { Driving/riding in car } \\
\text { TV/video viewing } \\
\text { Video games } \\
\text { Total Sitting minutes } \\
\text { Computer/Internet use for leisure } \\
\text { Reading } \\
\text { Sitting and talking with friends or } \\
\text { listening to music } \\
\text { Talking on phone }\end{array}$ & Age, gender, education, income & $\begin{array}{l}\text { Neighbourhood walkability } \\
\text { index, neighbourhood } \\
\text { income }\end{array}$ & Child living at home & 0.90 \\
\hline
\end{tabular}


Table 1 Overview of study characteristics (Continued)

\begin{tabular}{|c|c|c|c|c|c|c|c|}
\hline Kouvonen [51] & $\begin{array}{l}38151 \text { adults } \\
17-64 \text { years } \\
20 \% \text { men }\end{array}$ & CS & Time spent sedentary & Work effort-reward balance & & & 0.95 \\
\hline $\begin{array}{l}\text { Kozey-Keadle } \\
{[44]}\end{array}$ & $\begin{array}{l}58 \text { adults } \\
20-60 \text { years } \\
67 \% \text { men }\end{array}$ & QEX & Time spent sedentary & $\begin{array}{l}\text { Exercise, intervention to decrease } \\
\text { sedentary behaviour }\end{array}$ & & & 0.64 \\
\hline Lee [85] & $\begin{array}{l}410 \text { women age }= \\
42.5(\mathrm{SD}=9.3)\end{array}$ & CS & $\begin{array}{l}\text { Time spent sitting in motor } \\
\text { vehicles Total sitting time }\end{array}$ & & $\begin{array}{l}\text { Pedestrian crossing aids, } \\
\text { sidewalk traffic buffers, traffic } \\
\text { control device, number of } \\
\text { path connections, posted } \\
\text { speed limits, neighbourhood } \\
\text { attractiveness, } \\
\text { neighbourhood safety }\end{array}$ & & 0.82 \\
\hline Lepp [46] & $\begin{array}{l}302 \text { adults } \\
44 \% \text { men }\end{array}$ & CS & Leisure sedentary activities & Cell phone use & & & 0.82 \\
\hline Li [42] & 131 women & CS & Time spent in TVSE & $\begin{array}{l}\text { Age, education, work status, lack } \\
\text { of PA, BMI, depressive symptoms, } \\
\text { Perceived stress, knowledge/beliefs }\end{array}$ & & $\begin{array}{l}\text { Marital status, number of children } \\
\text { in the household, family } \\
\text { functioning }\end{array}$ & 0.95 \\
\hline Mabry [52] & $\begin{array}{l}10 \text { adults } \\
50 \% \text { men }\end{array}$ & Q & $\begin{array}{l}\text { Barriers to reduce prolonged } \\
\text { sitting }\end{array}$ & $\begin{array}{l}\text { Lack of motivation, knowledge/ } \\
\text { beliefs }\end{array}$ & Weather, access to facilities & $\begin{array}{l}\text { Social norms and community } \\
\text { participation }\end{array}$ & 0.80 \\
\hline Menai [41] & $\begin{array}{l}2841 \text { adults age } \\
57.3+/-5.0 \text { years } \\
38.3 \% \text { men }\end{array}$ & $\mathrm{L}$ & $\begin{array}{l}\text { Total leisure SB, Leisure TV } \\
\text { viewing, leisure computer use, } \\
\text { leisure reading, occupational } \\
\text { sitting, domestic sitting }\end{array}$ & $\begin{array}{l}\text { PA (leisure, walking, gardening, } \\
\text { swimming, biking, occupational, } \\
\text { domestic) }\end{array}$ & & Working status: retirement status & 0.88 \\
\hline Munir [66] & $\begin{array}{l}4436 \text { adults } \\
\text { Age from }<24 \text { to } \\
>55 \text { years } \\
44 \% \text { men }\end{array}$ & CS & Occupational sitting & $\begin{array}{l}\text { Age, BMl, PA levels, education, } \\
\text { job grade }\end{array}$ & & $\begin{array}{l}\text { Married/cohabitating, } \\
\text { dependents, work engagement, } \\
\text { job demands, job performance }\end{array}$ & 0.84 \\
\hline Oliver [18] & $\begin{array}{l}2033 \text { adults } 20-65 \\
\text { years } 43 \% \text { male }\end{array}$ & CS & Occupational sitting time & & $\begin{array}{l}\text { Neighbourhood level social } \\
\text { deprivation }\end{array}$ & & 0.76 \\
\hline Parry [76] & 22-59 years & CS & Time spent sedentary ${ }^{\circ}$ & & & Workdays vs. non-workdays & 0.90 \\
\hline Pomerleau [28] & $\begin{array}{l}6461 \text { adults } \\
19-65 \text { years }\end{array}$ & CS & Leisure time spent sedentary & $\begin{array}{l}\text { Education, income, smoking, } \\
\text { alcohol, vegetables intake }\end{array}$ & Rural vs. urban setting & & 0.68 \\
\hline Proper [49] & $\begin{array}{l}2650 \text { adults } \\
20-65 \text { years } \\
48 \% \text { men }\end{array}$ & $\mathrm{O}$ & $\begin{array}{l}\text { Sitting time on weekdays } \\
\text { Sitting time on weekend days } \\
\text { Sitting in leisure time }\end{array}$ & $\begin{array}{l}\text { Age, gender, education, } \\
\text { household income, total PA, } \\
\text { working hours }\end{array}$ & Neighbourhood SES & & 0.86 \\
\hline Rhodes [72] & $\begin{array}{l}206 \text { adults } \\
\text { Mean age }=54 \\
(S D=18.6) \\
51 \% \text { men } \\
174 \text { students } \\
\text { Mean age }=22 \\
(S D=13.2) \\
26 \% \text { men }\end{array}$ & CS & $\begin{array}{l}\text { TV Viewing } \\
\text { Computer-Use } \\
\text { Reading/Music } \\
\text { Socializing }\end{array}$ & $\begin{array}{l}\text { Attitude, intention, perceived } \\
\text { behaviour control, subjective } \\
\text { norm }\end{array}$ & & & 0.64 \\
\hline
\end{tabular}

8


Table 1 Overview of study characteristics (Continued)

\begin{tabular}{|c|c|c|c|c|c|c|c|}
\hline Saidj [17] & $\begin{array}{l}2308 \text { adults } 18-69 \\
\text { years } 46 \% \text { men }\end{array}$ & $C S / P$ & Leisure time sitting & & $\begin{array}{l}\text { Habitat type (apartment } \\
\text { versus house) and habitat } \\
\text { size (surface area) }\end{array}$ & $\begin{array}{l}\text { Household size (number of } \\
\text { occupants) }\end{array}$ & 0.76 \\
\hline Saidj [53] & $\begin{array}{l}35444 \text { adults } \\
44.5 \pm 13.0 \text { years } \\
79 \% \text { women }\end{array}$ & CS & $\begin{array}{l}\text { Domain-specific sitting time } \\
\text { (work, transport, leisure) }\end{array}$ & $\begin{array}{l}\text { Occupation type, perceptions } \\
\text { towards PA, age, gender, } \\
\text { education }\end{array}$ & & Workdays versus non-workdays & 0.84 \\
\hline Salmon [47] & $\begin{array}{l}1332 \text { adults } \\
>18 \text { years } \\
45 \% \text { men }\end{array}$ & CS & $\begin{array}{l}\text { Time spent sedentary } \\
\text { TV Viewing } \\
\text { Reading } \\
\text { Sitting Socializing }\end{array}$ & $\begin{array}{l}\text { Age, gender, lack of time to be } \\
\text { active, enjoyment of PA, } \\
\text { preference, tiredness, Injury, } \\
\text { disability }\end{array}$ & $\begin{array}{l}\text { Sidewalks, air or noise } \\
\text { pollution, weather } \\
\text { (perceived as a barrier), } \\
\text { safety, no access to facilities }\end{array}$ & $\begin{array}{l}\text { Family commitments, work } \\
\text { commitments }\end{array}$ & 0.8 \\
\hline Seguin [25] & $\begin{array}{l}92234 \text { women } \\
50-79 \text { years }\end{array}$ & $P$ & Time spent sedentary & $\begin{array}{l}\text { Age, education, ethnicity, } \\
\text { perceived health, physical } \\
\text { function, previous fall, BMl, } \\
\text { chronic diseases, hormone use, } \\
\text { medication, alcohol intake, levels } \\
\text { of PA, smoking }\end{array}$ & & Marital status & 0.8 \\
\hline Stamatakis [80] & $\begin{array}{l}7940 \text { adults } \\
\text { Mean age }=47 \\
(\mathrm{SD}=18.2) \\
44 \% \text { men }\end{array}$ & CS & Time spent in TVSE & Education, household income & Neighbourhood deprivation & Social class & 0.95 \\
\hline Stamatakis [79] & $\begin{array}{l}60404 \text { adults } \\
\geq 45 \text { years } \\
46 \% \text { men }\end{array}$ & CS & $\begin{array}{l}\text { Total sitting time } \\
\text { TV viewing } \\
\text { Computer time } \\
\text { Driving }\end{array}$ & $\begin{array}{l}\text { Education, annual household } \\
\text { income }\end{array}$ & $\begin{array}{l}\text { Area-level index of socio- } \\
\text { economic advantage }\end{array}$ & & 0.95 \\
\hline Stamatakis [22] & 2289 adults & CS & $\begin{array}{l}\text { TV viewing time } \\
\text { Sitting time in work } \\
\text { Sitting time outside work }\end{array}$ & $\begin{array}{l}\text { Household income, social class, } \\
\text { educational attainment, overall } \\
\text { socioeconomic position score }\end{array}$ & Area deprivation score & & 0.91 \\
\hline Storgaard [12] & $\begin{array}{l}48192 \text { adults } \\
44 \% \text { men }\end{array}$ & CS & Leisure time spent sedentary & Education, employment status & Density of green spaces & & 0.91 \\
\hline Strong [84] & $\begin{array}{l}1374 \text { adults mean } \\
\text { age }=45 \\
(\mathrm{SD}=12.9) \\
25 \% \text { men }\end{array}$ & CS & TV viewing & & $\begin{array}{l}\text { Neighbourhood problems } \\
\text { neighbourhood conditions }\end{array}$ & & 0.81 \\
\hline Sugiyama [34] & $\begin{array}{l}2224 \text { adults } \\
20-65 \text { years } \\
37 \% \text { men }\end{array}$ & CS & TV Viewing & $\begin{array}{l}\text { Age, education working status, } \\
\text { income, BMl, leisure time PA }\end{array}$ & $\begin{array}{l}\text { Neighbourhood SES, } \\
\text { neighbourhood walkability }\end{array}$ & & 0.91 \\
\hline Sugiyama [61] & $\begin{array}{l}2046 \text { adults } \\
20-65 \text { years } \\
36 \% \text { men }\end{array}$ & CS & $\begin{array}{l}\text { Time spent in other sedentary } \\
\text { behaviours (except TV viewing) }\end{array}$ & Time spent watching TV & & & 0.95 \\
\hline Sugiyama [65] & $\begin{array}{l}1408 \text { adults } \\
20-65 \text { years } \\
38 \% \text { men }\end{array}$ & CS & Time spent watching TV & BMI & & & 0.95 \\
\hline Sugiyama [77] & $\begin{array}{l}74788 \text { adults }>18 \text { years } \\
48 \% \text { men }\end{array}$ & $P$ & Prolonged time in car & $\begin{array}{l}\text { Age, work status, household } \\
\text { income, car ownership }\end{array}$ & Suburb, vicinity to CDD & Household composition & 0.68 \\
\hline
\end{tabular}


Table 1 Overview of study characteristics (Continued)

\begin{tabular}{|c|c|c|c|c|c|c|c|}
\hline Teychenne [62] & $\begin{array}{l}1554 \text { women } \\
18-65 \text { years }\end{array}$ & CS & TV Viewing & $\begin{array}{l}\text { Education, enjoyment of TV, } \\
\text { preference for sedentary } \\
\text { behaviour, stress and depressive } \\
\text { symptoms }\end{array}$ & $\begin{array}{l}\text { Neighbourhood safety, } \\
\text { neighbourhood aesthetic, } \\
\text { distance to places of } \\
\text { interest, distance to } \\
\text { physical activity facilities }\end{array}$ & $\begin{array}{l}\text { Social cohesion, social } \\
\text { participation, social support }\end{array}$ & 0.92 \\
\hline Thorp [75] & $\begin{array}{l}193 \text { adults } \\
34 \% \text { men }\end{array}$ & CS & Time spent sedentary $^{\circ}$ & Type of work & & Workdays vs. Non-workdays & 0. \\
\hline Touvier [78] & $\begin{array}{l}1389 \text { adults } \\
45-60 \text { years } \\
50 \% \text { men }\end{array}$ & $P$ & TV Viewing & Retirement & & & \\
\hline $\begin{array}{l}\text { Uijtdewilligen } \\
\text { [27] }\end{array}$ & $\begin{array}{l}11676 \text { adults, women } \\
\text { only }\end{array}$ & $P$ & $\begin{array}{l}\text { Time spent sitting at the weekend } \\
\text { and time spent sitting on weekdays }\end{array}$ & $\begin{array}{l}\text { BMI, country of birth, highest } \\
\text { educational qualification, } \\
\text { physical activity levels, smoking, } \\
\text { alcohol consumption, stress } \\
\text { levels, occupational status }\end{array}$ & Area of residence & $\begin{array}{l}\text { Number of children in the } \\
\text { household, marital status, work } \\
\text { commitment }\end{array}$ & \\
\hline $\begin{array}{l}\text { Uijtdewilligen } \\
\text { [71] }\end{array}$ & $\begin{array}{l}475 \text { from } 13 \text { to } \\
42 \text { years old } \\
47 \% \text { men }\end{array}$ & L & $\begin{array}{l}\text { Screen time: TV during leisure on } \\
\text { week or weekend days and time } \\
\text { spent behind computer during } \\
\text { leisure during week and weekend } \\
\text { days (h/week) }\end{array}$ & & & $\begin{array}{l}\text { Daily hassles (like conflicts with } \\
\text { colleagues, misbehaving } \\
\text { Children and being displeased about } \\
\text { personal appearance, and being } \\
\text { laughed at,...) } \\
\text { Life events (health, work, home/family, } \\
\text { personal/social relations, finance) }\end{array}$ & \\
\hline $\begin{array}{l}\text { Vandelanotte } \\
{[36]}\end{array}$ & $\begin{array}{l}2532 \text { adults } \\
20-65 \text { years } \\
39 \% \text { men }\end{array}$ & CS & $\begin{array}{l}\text { Leisure time internet and } \\
\text { computer use }\end{array}$ & $\begin{array}{l}\text { BMI, Other leisure time sedentary } \\
\text { behaviour (except TVSE) }\end{array}$ & & & \\
\hline Van Dyck [82] & $\begin{array}{l}1200 \text { adults } \\
20-65 \text { years } \\
47 \% \text { men }\end{array}$ & CS & Time spent sedentary $^{\circ}$ & $\begin{array}{l}\text { Age, gender, education, } \\
\text { employment status, occupation }\end{array}$ & $\begin{array}{l}\text { Neighbourhood walkability } \\
\text { index, neighbourhood SES }\end{array}$ & Living situation & \\
\hline Van Dyck [55] & $\begin{array}{l}419 \text { adults } \\
20-65 \text { years } \\
47 \% \text { men }\end{array}$ & CS & $\begin{array}{l}\text { TV ViewingLeisure time internet } \\
\text { use }\end{array}$ & $\begin{array}{l}\text { Age, gender, education, } \\
\text { employment status, BMl, pros } \\
\text { reducing TV viewing, cons } \\
\text { reducing TV viewing, self-efficacy } \\
\text { reducing TV viewing, pros } \\
\text { reducing internet use, cons } \\
\text { reducing internet use }\end{array}$ & $\begin{array}{l}\text { Number of PCs, number of } \\
\text { TVS, size of the largest TV } \\
\text { set }\end{array}$ & $\begin{array}{l}\text { Family social norm TV viewing, friends } \\
\text { norm TV viewing, family social norm } \\
\text { internet use }\end{array}$ & 0.9 \\
\hline Van Dyck [60] & $\begin{array}{l}6014 \text { adults } \\
20-65 \text { years } \\
44 \% \text { men }\end{array}$ & CS & $\begin{array}{l}\text { Overall sitting time } \\
\text { Motorized transport time }\end{array}$ & $\begin{array}{l}\text { Age, gender, education, having } \\
\text { a drivers licence, BMl }\end{array}$ & $\begin{array}{l}\text { Not many cul-de-sacs, not many } \\
\text { barriers in neighbourhood, } \\
\text { aesthetics, street, connectivity, } \\
\text { walking and cycling facilities, } \\
\text { access to services, proximity } \\
\text { to destinations, number of } \\
\text { different type of destinations } \\
\text { within } 20 \text { min walk from } \\
\text { home, parking difficult near } \\
\text { local shopping area, traffic } \\
\text { safety, crime safety, residential } \\
\text { density }\end{array}$ & Living with a partner & \\
\hline
\end{tabular}

Daily hassles (like conflicts with

reducing TV viewing, self-efficacy

reducing TV viewing, pros

reducing internet use

Age, gender, education, having

barriers in neighbourhood

0.95

safety, crime safety, residentia 
Table 1 Overview of study characteristics (Continued)

\begin{tabular}{|c|c|c|c|c|c|c|c|}
\hline Van Holle [16] & $\begin{array}{l}2839 \text { adults } 55-65 \\
\text { years } 52 \% \text { men }\end{array}$ & CS & $\begin{array}{l}\text { Sitting time during the weekend } \\
\text { days }\end{array}$ & & $\begin{array}{l}\text { Social trust and cohesion, } \\
\text { personal safety, aesthetics, } \\
\text { mean destination score, } \\
\text { number of TVs in the house }\end{array}$ & $\begin{array}{l}\text { Social participation, social support } \\
\text { from friends or colleagues ( }\end{array}$ & 0.80 \\
\hline $\begin{array}{l}\text { Van Uffelen } \\
\text { [24] }\end{array}$ & $\begin{array}{l}8920 \text { women } \\
25-30 \text { years } \\
11018 \text { women } \\
50-55 \text { years }\end{array}$ & CS & Sitting time & $\begin{array}{l}\text { Education, income, studying, } \\
\text { occupation, country of birth, } \\
\text { alcohol intake, levels of PA, } \\
\text { passive leisure activities, poor } \\
\text { sleeping, smoking, BMl, chronic } \\
\text { conditions, stiff/painful joints }\end{array}$ & Area of residence & $\begin{array}{l}\text { Marital status, number of children, } \\
\text { caring for family members }\end{array}$ & 0.90 \\
\hline $\begin{array}{l}\text { Wallmann- } \\
\text { Sperlich [10] }\end{array}$ & $\begin{array}{l}2000 \text { adults } \\
\text { Mean age }=49,3 \\
(\mathrm{SD}=17,6) \\
48 \% \text { men }\end{array}$ & CS & Sitting time & Age, gender, education, income & $\begin{array}{l}\text { Type of residence, aesthetics, } \\
\text { access to park and } \\
\text { recreational facilities, distance } \\
\text { to local facilities, public } \\
\text { transport infrastructure, } \\
\text { neighbourhood safety -traffic } \\
\text { and crime }\end{array}$ & & 0.90 \\
\hline Wilson [37] & $\begin{array}{l}68 \text { adults } \\
47 \% \text { men }\end{array}$ & CS & $\begin{array}{l}\text { Time spent sedentary } \\
\text { TV Viewing }\end{array}$ & $\begin{array}{l}\text { Age, education, family income, } \\
\text { employment type, levels of PA, } \\
\text { anthropometrics }\end{array}$ & & & 0.41 \\
\hline Xie [23] & $\begin{array}{l}3016 \text { adults } \\
\geq 18 \text { years } \\
46 \% \text { men }\end{array}$ & CS & TV Viewing & $\begin{array}{l}\text { Age, gender, employment, } \\
\text { education, BMI, smoking, alcohol } \\
\text { intake, vigorous PA }\end{array}$ & & Marital status & 0.95 \\
\hline Zolnk [15] & $\begin{array}{l}2943 \text { households } \\
25-65 \text { years }\end{array}$ & CS & Private vehicle commuting time & Income, occupation, gender & $\begin{array}{l}\text { Degree of centredness } \\
\text { (urban/rural subway) }\end{array}$ & & 0.68 \\
\hline
\end{tabular}

$B M I$ body mass index, $C B D$ central business district

Study design: CS cross sectional, $L$ longitudinal, $O$ observational, $P$ prospective, $Q$ qualitative, $Q E X$ quasi-experimental

anly study to investigate policy factors: worksite physical activity policy, work place health promotion programme 


\section{Individual correlates}

\section{Type of individual-level factors}

Of the 74 studies included, 62 examined the relationship between sedentary behaviours and individual factors. Four categories of factors were identified: behavioural (lifestyle, physical activity and sedentary habits $(n=30)$ ), physical, biological and genetic (age, gender, body composition, health status and medication $(n=26))$, psychological (stress and depressive symptoms, attitudes and perceptions $(n=25))$ and socioeconomic status (educational levels, employment/occupational status and income $(n=23))$. All individual factors were assessed using self-report questionnaires apart from some of the physical, biological and genetic factors (e.g., body mass index and heritability) that were measured objectively. Table 2 provides a detailed overview.

\section{Behavioural factors}

Thirty studies examined lifestyle factors: alcohol consumption $(n=7)$, food intake $(n=5)$, smoking $(n=7)$ and physical activity $(n=17)$. Alcohol consumption was found to be unrelated to sedentary behaviours in the three of the five studies [23-25] that examined its correlation as an individual factor. The remaining two found it to be positively associated with time spent sedentary in transport (driving) [26] and to overall weekend sedentary time [27]. Similarly when combined with diet, it was shown to have a positive association $[28,29]$ to TVSE and overall leisure sitting time. Four studies investigating food cravings, snacking and high calorie snacking found that sedentariness was highly associated with all four [27, 30-32]. In six of the seven studies investigating smoking, it was shown to be positively associated with sedentary time as measured by TVSE, time spent driving and total sitting time [24-29, 33]. In terms of physical activity, 18 studies examined its relationship with sedentary behaviour. The majority looked at overall physical activity levels $(n=11)$ and found there to be an inverse association $[24,25,31,34-38]$. This was also the case for the three studies that explored levels of physical activity outside of work-time [34, 39, 40]. One study examined the association between retirement and physical activity levels in a number of different sedentary domains (TVSE, leisure reading, occupational sitting and domestic sitting) and reported no correlation between it and any of the domains [41]. Similarly, a lack of physical activity [42], vigorous physical activity [23] and total time exercising [43] were not significantly associated with sedentariness. One study conducted a four-armed randomised trial investigating whether (i) supervised exercise, (ii) supervised exercise with advice to decrease sedentary time or (iii) advice to decrease sedentary time and increase non-exercising physical activity levels [44] would change total sedentary time, as measured by an inclinometer. Results revealed that structured exercise was ineffective; only those in the group that were given advice to try and change their sedentary behaviours and increased their daily physical activity levels showed a significant change in total sedentary time. Finally, sedentary habits such as TV viewing and cell phone use (gaming and suing wifi) were found to be positively associated with total time spent sitting $[24,34,45]$ and TVSE $[36,46]$.

\section{Physical, biological and genetic factors}

Twenty-six studies investigated physical, biological or genetic factors with all of them evaluating age as a correlate of sedentary behaviour. Fourteen of twenty studies supported a positive relationship between age and sedentary behaviours (the older the person, the more sedentary). Eight studies looked at total sitting time, five of which positively correlated with age. Overall the results were mixed between positive correlations and no significant correlations. No studies reported a negative correlation and furthermore findings could not be differentiated by their sedentary measurements.

Gender was investigated in 19 studies. Ten reported the female gender to be inversely related to sedentariness $[23,37,43,47-53]$ with two reporting males to be more sedentary when associated with total time spent in front of a computer screen and overall leisure sitting time $[53,54]$. The remainder found little or no association with either gender [12, 33, 45, 55-57]. The majority of studies reported gender differences defined sedentariness as total sitting time [43, 47, 49-51] or TVSE [23, 48] with one using accelerometry [37], one using heart rate [56] and one reporting barriers to changing sedentary time in Oman via semi-structured interviews [52]. Four studies reported the male gender to be positively associated with sitting time in transport [53, 58-60].

The relationship between sedentary behaviour and body mass index (BMI) was evaluated in 25 papers, the majority of which investigated its association with leisure screen time. Seventeen of these studies [23, 25, 29, 31, 32, 34-36, $49,55,56,60-65]$ reported a positive relationship with the remainder showing no correlation. Nine studies examined this association using total sitting time and two used accelerometers as an objective measure [30, 37] and one heart rate [56]. The two studies that used accelerometry reported no significant relationship between BMI and total sedentary time while the majority of the remaining studies showed that the higher the BMI the higher the level of sedentariness. Two studies looked at the association between occupational sitting and BMI and reported a positive association $[54,66]$. Overall results suggest that there is a strong relationship between increased BMI and higher level of sedentary behaviours.

Chronic diseases (e.g., diabetes, cardiovascular disease) were shown to have a positive relationship with sedentary 
Table 2 Individual correlates of sedentary behaviours in adults

\begin{tabular}{|c|c|c|c|c|c|c|}
\hline \multicolumn{7}{|c|}{ Individual Correlates of Sedentary Behaviour in Adults (18-65 years) } \\
\hline Factors ( $n=$ total studies) & Total screen time & Leisure screen time & Transport sitting time & Total sitting time & Leisure sitting time & Total Objective SB \\
\hline \multicolumn{7}{|l|}{ Behavioural } \\
\hline Alcohol consumption $(n=5)$ & & $\operatorname{nr}[23]$ & $+[26]$ & $\begin{array}{l}+[27]^{\mathrm{W}} \\
\operatorname{nr}[24]^{\mathrm{W}},[25]^{\mathrm{W}}\end{array}$ & & \\
\hline Alcohol and diet $(n=1)$ & & & & & $+[28]$ & \\
\hline Food cravings $(n=1)$ & & & & $+[30]^{W}$ & & $+[30]^{\mathrm{W}}$ \\
\hline High calorie snacking $(n=4)$ & & $+[31],[32],[40]$ & $+[26]$ & & & \\
\hline Lifestyle $(n=1)$ & & $+[29]$ & & & & \\
\hline Smoking $(n=7)$ & & $\begin{array}{l}+[33] \\
\operatorname{nr}[23]\end{array}$ & $+[26]$ & $+[24]^{\mathrm{W}},[25]^{\mathrm{W}},[27]^{\mathrm{W}}$ & $+[28]$ & \\
\hline Lack of PA $(n=1)$ & & $\mathrm{nr}[42]^{\mathrm{W}}$ & & & & \\
\hline PA (vigorous) $(n=2)$ & & $\operatorname{nr}[23]$ & & & & $\mathrm{nr}[44]$ \\
\hline PA levels $(n=11)$ & $\mathrm{nr}[41]$ & $\begin{array}{l}-[31]^{\mathrm{W}},[34]^{\mathrm{W}},[35]^{\mathrm{M}},[36] \\
\operatorname{nr}[73],[41]\end{array}$ & & $\begin{array}{l}+[40] \\
-[24]^{\mathrm{W}},[25]^{\mathrm{W}}[37],[27]^{\mathrm{W}}\end{array}$ & $\mathrm{nr}[41]$ & $-[37]$ \\
\hline PA outside work $(n=2)$ & & $-[34]^{W},[39]$ & & & & $-[39]$ \\
\hline Total time in exercise $(n=1)$ & & & & $\operatorname{nr}[43]^{\mathrm{M}}$ & & \\
\hline Poor sleeping habits $(n=2)$ & & & $+[26]$ & $-[24]^{\mathrm{W}}$ & & \\
\hline Sedentary habits $(n=2)$ & & $+[36]$ & & $+[45]$ & & $+[45]$ \\
\hline Cell phone use $(n=1)$ & & $+[46]$ & & & & \\
\hline TV viewing time $(n=1)$ & & & & $+[61]$ & & \\
\hline \multicolumn{7}{|l|}{ Physical/Biological/Genetic } \\
\hline Age $(n=20)$ & $\mathrm{nr}[53]$ & $\begin{array}{l}+[23],[32],[63],[55],[48],[48],[49]]^{\mathrm{W}},[34]^{\mathrm{W}} \\
\operatorname{nr}[59],[42]^{\mathrm{W}},[9],[31]^{\mathrm{W}},[34]^{\mathrm{M}}\end{array}$ & $+[48]$ & $\begin{array}{l}+[30]^{W}{ }^{\prime}[49]^{W},[50],[10],[60] \\
\operatorname{nr}[25]^{W^{W}},[47],[37],\end{array}$ & $+[53]$ & $\begin{array}{l}+[30]^{\mathrm{W}} \\
\operatorname{nr}[37]\end{array}$ \\
\hline Gender $(n=19)$ & & $\begin{array}{l}-[23],[48] \\
\text { nr [55], [57] }\end{array}$ & $\begin{array}{l}+[53],[15],[59], \\
{[60]}\end{array}$ & $\begin{array}{l}+[54]^{\mathrm{M} \text { occ }} \\
-[47],[50],[49],[51],[43],[52] \\
\operatorname{nr}[33],[12]\end{array}$ & $+[53]$ & $\begin{array}{l}-[37] \\
\operatorname{nr}[45],[56]\end{array}$ \\
\hline $\mathrm{BMI}(n=25)$ & & $\begin{array}{l}+[23],[55],[29],[61],[63],[31]^{\mathrm{W}},[49],[34], \\
{[35]^{\mathrm{M}},[32],[65]^{\mathrm{M}},[36],[62]^{\mathrm{W}}} \\
\operatorname{nr}[42]^{\mathrm{W}}\end{array}$ & $+[48]$ & $\begin{array}{l}+[25]^{\mathrm{W}},[48],[64]^{\mathrm{W}},[27]^{\mathrm{W}}[54]^{\circ c c},[40]^{\circ c c} \\
\text { nr [30] }]^{\mathrm{W}},[24]^{\mathrm{W}},[37],[66]^{\circ c c}\end{array}$ & & $\begin{array}{l}+[56] \\
\operatorname{nr}[30]^{\mathrm{W}},[37]\end{array}$ \\
\hline Chronic diseases $(n=4)$ & & & & $\begin{array}{l}+[25]^{\mathrm{W}},[32],[67]^{\mathrm{M}} \\
\operatorname{nr}[24]^{\mathrm{W}^{\mathrm{N}}}\end{array}$ & & \\
\hline Disability, Illness, Injury $(n=5)$ & & & $+[26]$ & $\operatorname{nr}[47], \operatorname{nr}[30]^{\mathrm{W}},[24]^{\mathrm{W}},[68]^{\mathrm{W}}$ & & $\mathrm{nr}[30]^{\mathrm{W}}$ \\
\hline Hormone use $(n=1)$ & & & & $+[25]^{\mathrm{W}}$ & & \\
\hline Medication $(n=1)$ & & & & $+[25]^{\mathrm{W}}$ & & \\
\hline Pregnancy $(n=1)$ & & & & & & $+[70]$ \\
\hline
\end{tabular}


Table 2 Individual correlates of sedentary behaviours in adults (Continued)

\begin{tabular}{|c|c|c|c|c|c|}
\hline Race $(n=3)$ & $\begin{array}{l}+[31]^{\mathrm{W}} \\
\operatorname{nr}[42]^{\mathrm{W}}\end{array}$ & & $+[25]^{W}$ & & \\
\hline Heritability $(n=1)$ & & & & & $+[69]$ \\
\hline \multicolumn{6}{|l|}{ Psychological } \\
\hline Attitude $(n=1)$ & & & $-[72]$ & & \\
\hline $\begin{array}{l}\text { Depressive symptoms, anxiety, } \\
\text { tension or stress }(n=7)\end{array}$ & $\begin{array}{l}+[42]^{\mathrm{W}},[57],[31]^{\mathrm{W}}[29] \\
\operatorname{nr}[62]^{W_{(m e d)}}\end{array}$ & $+[26]$ & $+[27]^{W}$ & & \\
\hline Enjoyment of TV $(n=1)$ & $+[62]^{\mathrm{W}}$ & & & & \\
\hline Intention $(n=3)$ & & & $\begin{array}{l}-[45],[72] \\
\mathrm{nr}[54]^{\text {occ }}\end{array}$ & & $-[45]$ \\
\hline Perceived behavioural control $(n=2)$ & & & $\operatorname{nr}[72],[54]^{\circ c c}$ & & \\
\hline Perceived health $(n=3)$ & $-[31]^{\mathrm{W}}$ & & $-[25]^{\mathrm{W}}$ & $\mathrm{nr}[53]$ & \\
\hline Perceived benefits of reducing SB $(n=2)$ & $-[55]$ & & $-[52]$ & & \\
\hline Preference $(n=2)$ & $\mathrm{nr}[62]^{\mathrm{W}(\mathrm{med})}$ & & $+[47]$ & & \\
\hline Subjective Norm $(n=2)$ & & & $\begin{array}{l}+[52] \\
\operatorname{nr}[72]\end{array}$ & & \\
\hline \multicolumn{6}{|l|}{ Socio-economic Status } \\
\hline Level of educational attainment $(n=22)$ & $\begin{array}{l}-[23],[31]^{\mathrm{W}},[42]^{\mathrm{W}}, \text { [55], [9], [48], [40], [22] } \\
\operatorname{nr}[63]\end{array}$ & $\operatorname{nr}[48]$ & 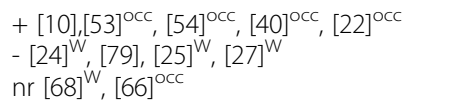 & $-[28]^{\mathrm{M}},[12]$ & $+[22]$ \\
\hline Employed $(n=7)$ & - [23], [31] $]^{\mathrm{W}},[63],[55],[9],[48]$ & & & $+[12]$ & \\
\hline Manual Employment $(n=4)$ & $+[73]$ & & $\begin{array}{l}+[38] \\
-[37]\end{array}$ & & $\begin{array}{l}+[22] \\
-[37]\end{array}$ \\
\hline Office work $(n=9)$ & - [40], [54], [22] & $+[46]$ & $\begin{array}{l}+[74],[38],[27]^{\mathrm{W}},[40]^{0 c c},[53,54]^{\circ c c},[22]^{\circ c c} \\
-[60],[67]^{\mathrm{M}}\end{array}$ & & $+[22],[75]$ \\
\hline Work vs non-work time $(n=5)$ & & $+[77]$ & $S[74]$ & & $\mathrm{S}$ [39], [76], [75] \\
\hline Full time versus part-time work $(n=3)$ & & S [27], [66], [74] & S [27], [66], [74] & & \\
\hline Change at work $(n=1)$ & & & S [68] & & \\
\hline Work commitment $(n=3)$ & & & $-[67]^{\mathrm{M}},[52]$ & $-[47]$ & \\
\hline Retirement $(n=3)$ & $+[41,78],[73]$ & & & $+[41]$ & \\
\hline Studying $(n=1)$ & & & $+[24]^{W}$ & & \\
\hline Household Income $(n=10)$ & $\begin{array}{l}-[48],[22],[40] \\
\operatorname{nr}[63]\end{array}$ & $+[77]$ & $\begin{array}{l}+[59],[80],[22]^{o c c},[40]^{\circ c c} \\
-[79]\end{array}$ & $-[28]^{\mathrm{M}}$ & $+[59],[22]$ \\
\hline Income $(n=8)$ & $\begin{array}{l}-[30]^{\mathrm{W}},[73] \\
\operatorname{nr}[9]\end{array}$ & & $\begin{array}{l}+[54]^{\mathrm{ccc}} \\
-[24]^{\mathrm{W}},[30]^{\mathrm{W}},[37] \\
\operatorname{nr}[10]\end{array}$ & & - [30] ${ }^{W_{,}}[37]$ \\
\hline
\end{tabular}

Note: Each result is reported as positive (+), negative $(-)$, or not related $(\mathrm{nr})$ for objective or self-reported/perceived individual measure. Significant associations only in subgroups are identified as men $\left({ }^{\mathrm{M}}\right)$, women $(\mathrm{M})$ occ refers to occupational time. $S$ refers to significant differences between groups. For one study [62], the studied factor was investigated as a mediator of the association between education and sedentary behaviour and identified as ("med) 
time in three of the four studies $[25,32,67]$ that included them. In none of the studies were they the primary factors being investigated. In contrast, illness, previous surgery, disability and injury were shown to have no significant correlation to total sitting time [24, 30, 47, 68]. One study investigated the role of heritability [69], one pregnancy [70], and another the role of hormone treatment and medication [25]. All three reported significant correlations with sedentariness.

\section{Psychological factors}

Fifteen studies included psychological factors. However few investigated more than one factor. Five studies investigated depressive symptoms and four found that symptoms of depression, anxiety and tension were positively related to total screen time [29, 31, 42, 57]. Similarly perceived stress levels $[26,27,42]$ and perceived tiredness [47] were also positively associated, whereas perceived health $[25,31]$ and perceived benefits of reducing sedentary behaviours [53-55] were found to be inversely associated with sedentariness as measured by occupational sitting, TVSE and total sitting time. One study investigating perception of personal appearance and content with body image found no relationship to sedentariness [71]. Rhodes et al. [72] investigated whether planned behaviour is related to sedentary behaviours. They found mixed results; attitude and intention were negatively correlated with sedentariness as measured by total sitting time while perceived control and norm were not. Conroy et al. [45] also reported that intention and habit in terms of regulating sedentary behaviour were negatively associated. Overall the limited available evidence is supportive for a positive relationship between perceived feelings of depression, stress and anxiety and TVSE and a negative relationship between sedentary behaviour and planned behaviour to overcome sedentariness.

\section{Socio-economic factors}

Twenty-two studies investigated educational levels and their relationship to sedentary behaviours. Nine examined TVSE, eleven used total sitting time or occupational sitting, two total leisure sitting time and one accelerometry for total sedentary time [22]. Of the nine that examined the correlation between educational levels and TVSE, eight reported significant inverse correlations $[9,22,23,31,40,42,48,55]$ and one reported no significant relationship [63]. In terms of total sitting time, five studies focused on occupational sitting as a domain of total sitting found there was a positive relationship with educational attainment $[10,22,40,53,54]$ whereas the studies that investigated total sitting time without classifying domains found it to be negatively correlated. The exception was total sitting time as measured by actigraphy; it was found to have a positive association with educational attainment [22].
Occupation and employment were explored as a potential factor in seventeen studies. In relation to TVSE, it was positively related to unemployment while the opposite was true for employment (negatively correlated) $[9,12,23,31,48,55,63]$. Storgaard et al. [12] investigated employment in relation to all leisure sitting time as opposed to just TVSE and reported it to be similar to TVSE; positively related to unemployment and negatively related to employment.

Type of employment was reported in some of the studies. Manual employment, investigated in four studies $[22,37,38,73]$ was positively correlated to sedentariness outside of work where sedentariness was measured as total sitting time both subjectively and objectively. In contrast, working in an office was more likely to result in less sedentary time outside of work. Chau et al. [38] and Jans et al. [74] reported increased total sitting time associated with working in an office. Thorp et al. [75] found call centre employees to be more sedentary during the working day than customer service workers. Five further studies exploring type of occupation showed that those in professional roles were more likely to have a higher level of occupation sitting than those in nonprofessional positions [22, 27, 40, 53, 54].

Five studies focused on whether sedentary behavior differed based on work and non-work time days. Overall, work days corresponded to more sedentary time [39, 74-77] and a greater amount of time spent in prolonged sitting, when compared to non-work days [75, 76]. Saidj et al. [53] reported the more sedentary the occupation type the higher the association with increased sedentary time in other domains (work, transport, leisure, screen time) during weekdays but not during the weekend.

Total sitting time, sitting time during work and traveling to and from work was significantly higher for full-time workers than for part-time workers [27, 66, 74]. Also, work commitments as barriers for physical activity were inversely associated with reported time spent sedentary $[47,52,67]$. Munir reported that vigor at work was associated with less occupational sitting in men and women, but the association was unclear across gender for absorption at work, dedication or job performance [66]. In women, change at work, return to study or new work was associated with an increase in total sitting [68].

Other factors relating to employment that were investigated were retirement and studying. From the retirement perspective, four studies found that retirement resulted in an increase in sedentary behaviour $[41,68,73,78]$. Retirement was associated with an increase in total $[68,78]$, leisure SB (screen, reading, total) and domestic sitting [41, 73]. Van Uffelen et al. [24] reported a significant increase in overall sitting time in women who were studying.

Sixteen studies examined the relationship between income and sedentary behaviours using various measures 
of sedentariness. One study reported a positive association with sitting time spent in transport [77] and three studies that focused on occupational sitting time [22, 40, 54] also found a positive relationship. Of the remaining studies, TVSE as a sedentary measure was used in seven studies, five of which reported a negative relationship [22, 30, 40, 48,73 ] while the remaining two showed no correlation $[9,63]$. Six studies examined income and its relationship to total sitting time $[10,24,30,37,59,79,80]$. Finally one study focused on leisure sitting time [28] and found it to be inversely related. In terms of total sitting time and its relationship to income, all studies but one [10] reported a relationship, mainly in a positive direction. Finally in terms of objectively measured total sedentary time and its relationship with household income, both Kozo et al. [59] and Stamatakis et al. [22] found a clear positively association.

\section{Interpersonal correlates}

\section{Type of interpersonal level factors}

The relationship between interpersonal factors and sedentary behaviours was examined in 22 studies, 14 were cross-sectional; 3 longitudinal, 3 prospective and two were qualitative. Two domains of interpersonal factors were identified, family-related (marital status, living arrangements, family functioning, number of children, family commitment $(n=17)$ ) and social factors (social norms, social interaction, cohesion, support and participation, sense of community; $(n=7))$. All interpersonal factors were assessed using self-administered or interviewadministered validated questionnaires. Table 3 provides detailed findings relating to interpersonal factors from the 22 identified papers.

\section{Family-related factors}

Eight studies investigated the relationship between "marital status" and sedentary behaviour. In Japanese adults, Ishii et al. [63] reported that unmarried subjects were likely (odds ratio [OR], 2.02; $95 \% \mathrm{CI}, 1.32-3.10$ ) to spend more time in TVSE than married subjects $(>14 \mathrm{~h} /$ week). Van Uffelen et al. [24] found sitting time to be significantly higher in single women [24] while van Dyck et al. [60] showed adults living with a partner [60] sat less. Another study conducted in Hong Kong reported contradictory findings. Xie et al. found TV viewing time to be higher in married persons [23].

No relationship was found between TV viewing and marital status in a group of low-income women [42], neither between occupational sitting and marital status in men or women [66]. Clark et al. [68] using a prospective study design investigated the relationship between life events and sedentary behaviour and found change in marital status was not associated with changes in total sitting [68].

In terms of "living arrangement", whether someone lived alone or with others was not associated with screen time [63] or TV viewing [9]. One study did however report that men living alone were more likely to watch TV for $4 \mathrm{~h}$ /day or more [48]. Only one study investigated the association between TV viewing time and lower "family functioning" (likert score) in a group of low-income women and found

Table 3 Interpersonal correlates of sedentary behaviour in adults

\begin{tabular}{|c|c|c|c|c|c|c|}
\hline \multicolumn{7}{|c|}{ Interpersonal correlates of Sedentary Behaviours in Adults (18-65 years) } \\
\hline Factors ( $n=$ total studies) & Total screen time & Leisure screen time & $\begin{array}{l}\text { Transport sitting } \\
\text { time }\end{array}$ & Total sitting time & $\begin{array}{l}\text { Leisure sitting } \\
\text { time }\end{array}$ & $\begin{array}{l}\text { Total Objective } \\
\text { SB }\end{array}$ \\
\hline \multicolumn{7}{|l|}{ Family } \\
\hline Marital status $(n=8)$ & & $\begin{array}{l}+[23] \\
-[63] \\
\operatorname{nr}[42]^{w}\end{array}$ & $\operatorname{nr}[60]$ & $\begin{array}{l}-[24]^{\mathrm{w}},[60] \\
\mathrm{nr}[27],[68],[66]^{\mathrm{occ}}\end{array}$ & & \\
\hline Living arrangements $(n=3)$ & & $\begin{array}{l}\operatorname{nr}[63],[9] \\
\text { S [48] }\end{array}$ & & & & \\
\hline Family functioning $(n=1)$ & & $-[42]^{w}$ & & & & \\
\hline Number of children $(n=8)$ & & $\begin{array}{l}-+[59],[53] \\
\operatorname{hr}[9]^{w},[42]^{w}\end{array}$ & $+[77],[59]$ & $-[24]^{\mathrm{w}}[59],[71],[68]^{\mathrm{b}}$ & $-[59]$ & - [59] \\
\hline Family commitment $(n=5)$ & & & & $\begin{array}{l}-[24]^{\mathrm{w}},[66]^{\mathrm{occ}} \\
+[67]^{\mathrm{M}},[52]\end{array}$ & $+[47]$ & \\
\hline \multicolumn{7}{|l|}{ Social factors } \\
\hline Social norms $(n=3)$ & & $+[55]$ & & $-[52]$ & $\mathrm{nr}[16]$ & \\
\hline $\begin{array}{l}\text { Social cohesion, interaction, } \\
\text { support and participation }(n=5)\end{array}$ & & $\begin{array}{l}-[62]^{\text {med }} \\
\text { nr [9], [62] }]^{\text {med }},[84],[71]\end{array}$ & & & $\mathrm{nr}[16]$ & \\
\hline Sense of community $(n=2)$ & & $\mathrm{nr}[9]$ & & $-[52]$ & & \\
\hline
\end{tabular}

Note: Each result is reported as positive (+), negative (-), or not related (nr) for objective or self-reported/perceived intrapersonal measure. Significant associations only in subgroups are identified as men $\left({ }^{\mathrm{M}}\right)$, women $\left({ }^{\mathrm{w}}\right) .{ }^{\mathrm{f}}$ refers to friends/colleagues support; ${ }^{\mathrm{b}}$ refer to birth of child; occ refers to occupational timerefers to occupational S refers to significant differences between groups. For one study [62], the studied factor was investigated as a mediator of the association between education and sedentary behaviour and identified as $\left({ }^{\text {med }}\right)$ 
a positive correlation $(r=0.28, p<0.01)$ that remained significant in a multivariable model including stress and depressive symptoms [42]. The impact of the "number of children" on sedentary behaviour as assessed by TV viewing time was not significant in Li's study in low-income women [42], nor was it significant in a longitudinal study by Ding [9]. In contrast, several authors found that that overall sitting time was lower with more children [24] or with the birth of child [24, 53, 68, 71]. Kozo et al. investigated several sub-types of sedentary behaviour and found that having no children was related to more TV/video viewing, computer/Internet use, sitting and talking with friends or listening to music, total sitting time or accelerometer-measured sedentary time [59]. Two studies found a positive association between number of children and transport sitting time [59, 77]. Family commitment defined as providing care for other members of the family was investigated in the Australian Longitudinal Study on Women's Health [24]. It revealed that women who cared for others spent less time sitting, particularly younger women. Similarly, having more dependents was associated with decreased occupational sitting time in men and women [66]. In contrast, Salmon et al. reported family commitments as a factor that decreased physical activity resulting in an increase in total sedentary time [47]. This finding was further supported by George et al. [67] and Mabry et al. [52] in their qualitative studies. They both reported family commitments as a barrier to decreasing sedentary time. Taken together, these results show that family-related factors show inconsistencies for their relationship with sedentariness.

\section{Social factors}

Six studies investigated other social factors such as social norms $[52,55]$, social cohesion, interaction, support and participation and sense of community $[9,16,52,62]$. Social norms were found to correlate with leisure screen time in one study. Although other factors were not significantly associated with sedentary behaviour they were significant mediators of the impact of education on this unhealthy behaviour [62]. No social factors were found to correlate with weekend sitting time with or without interaction with retirement status [16] Finally a study by Uijtdewilligen et al. [27] investigating daily hassles found no significant association with screen time [71].

\section{Environmental correlates}

\section{Type of environmental level factors}

Of the 74 studies included, 33 considered environmental exposure. Environmental exposures/resources is categorised under five domains, four of which are previously proposed in other publications [81]; physical environment, services available in the environment, socio-demographic environment, neighbourhood safety and the additional domain of the home/work indoor environment. Twenty four studies account for factors related to the physical environment, fourteen thirteen considered variables of the socio-demographic environment (neighbourhood socioeconomic status, deprivation), nine examined factors relating to neighbourhood safety and eight investigated service environment variables (recreation facilities, access to services, proximity of destinations). Finally, four studies considered the indoor environment at home or at work (furniture, number of TVs/PCs).

In term of measurement, self-reported/perceived and objective assessments of environmental characteristics were quite equally distributed across the studies. The most commonly used objective measure of environmental factors was GIS techniques and composite environmental measures (e.g., neighbourhood walkability index, neighbourhood deprivation index). Table 4 provides a detailed account of the included studies, the investigated environmental variables and measurement tools.

\section{Physical environment}

Mixed results were observed regarding the effect of living in a rural or urban area, dependent on the type of sedentary behaviour considered. Van Uffelen et al. [24] and Uijtdewilligen et al. [27] found that living in an urban area resulted in higher total sitting time among women compared to those living in a rural town. Likewise, Clark et al. [48] reported that living in a regional city outside of the state capitals was associated with an increased likelihood of watching two or more hours of television per day. Pomerleau et al. [28] found similar associations between urban area and sedentary behaviour during leisure time but this was dependent on nationality; Estonian men and Lithuanian women living in towns and cities were more sedentary than their rural counterparts however the opposite is true for Latvian men and women. Two studies showed that living in a rural area was positively associated with transport sitting time [15, 77].

In terms of aesthetics, only one of six studies reported a significant negative association between neighbourhood aesthetics and overall sitting time [60] while five studies reported no associations with sedentary behaviours. Considering green spaces, an increase in the density [12] and a greater proximity [14] were associated with a decrease in sedentary behaviour time. Five studies considered neighbourhood walkability showing mixed results. Three of these reported a negative association with sedentary behaviours. Sugiyama et al. [34] found that women in high-walkability neighbourhoods spend less time watching TV than their counterpart in moderate or low walkability neighbourhoods. Similarly, Kozo et al. [59] showed neighbourhoods with high walkability decrease total sitting time among both men and women. 
Table 4 Environmental correlates of sedentary behaviours in adults

Environmental Correlates of Sedentary Behaviours in Adults (18-65 years)

Home/work indoor environment

Number of PCs at home $(n=1)$

Leisure screen time

Transport sitting time

Total sitting time

Leisure sitting time

Total Objective SB

Number of TVs at home $(n=2)$

nr [55]

$+[55]$

$\operatorname{nr}[16]$

Size of the largest TV set $(n=1)$

$+[86]^{b}$

Lockers for clothes at work $(n=1)$

$+[86]^{b}$

$+[86]^{b}$

Safe bike storage at work $(n=1)$

Habitat surface area $(n=1)$

Habitat type (apartment vs. house) $(n=1)$

$-[17]$

ns [17]

ns [17]

Physical environment

Type of residence $(n=1)$

Not many cul-de-sacs/barriers in

neighbourhood $(n=1)$

nr [10]

nr [60]

Aesthetics/attractiveness $(n=6)$

Proximity/density of green spaces $(n=2)$

Neighbourhood walkability $(n=5)$

Walking and/or cycling facilities $(n=4)$

Street connectivity $(n=2)$

Land -use mix $(n=1)$

Traffic safety $(n=4)$

nr [9], [11]

$\operatorname{nr}[9],[62]^{\mathrm{W}(\mathrm{med})}$

$-[34]^{\mathrm{WaO}},[59]^{\mathrm{aO}}$

[9] nwr ${ }^{\mathrm{aO}}$

$-[11]$

nr [9]

Air/noise pollution $(n=1)$

Weather as a barrier $(n=3)$

Season $(n=1)$

Living outside State Capital $(n=1)$

Living rurally (vs. urban) $(n=5)$

Region $(n=1)$

$+[48]^{\circ}$
$+[85]^{\mathrm{W}}$

$-[60]^{a}$

nr [10]

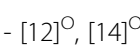

$+[82]^{\mathrm{aO}}$

$-[59]^{\mathrm{aO}}$

nr $[8]^{\mathrm{WaO}}$

$\operatorname{nr}[60]^{\mathrm{a}}$, [47]

$\operatorname{nr}[60]^{\mathrm{a}}$

$-[83]^{\mathrm{u} O}$

nr $[60]^{\mathrm{a}}$

$-[83]^{\mathrm{u} O}$

nr [60 $]^{\mathrm{a}}$

$+[10]^{W}$
$-[60]^{W a}$

$+[47]$

+ [47], [67], [52]

$+[15]^{\circ},[77]^{\circ}$

$-[24]^{w},[27]^{w O}$

$+u[28]$ $+[8]^{\mathrm{ja}}$

nr [59] $\operatorname{nr}[13]^{\circ}$

$\operatorname{nr}[13]^{\circ}$ 
Table 4 Environmental correlates of sedentary behaviours in adults (Continued)

\begin{tabular}{|c|c|c|c|c|c|}
\hline \multicolumn{6}{|l|}{ Services available in the environment } \\
\hline Access to services $(n=4)$ & $\operatorname{nr}[11]$ & $\mathrm{nr}[60]^{\mathrm{a}}$ & $\begin{array}{l}-[60]^{\mathrm{ma}} \\
\mathrm{nr}[47]\end{array}$ & $\operatorname{nr}[16]$ & \\
\hline Proximity/distance to destinations $(n=3)$ & $\mathrm{nr}[62]^{\mathrm{W}(\mathrm{med}) \mathrm{a}}$ & $\mathrm{nr}[60]^{\mathrm{a}}$ & $\begin{array}{l}-[60]^{\mathrm{ma}} \\
\mathrm{nr}[10]\end{array}$ & & \\
\hline Access to recreation facilities $(n=4)$ & $\operatorname{nr}[11]$ & & $\begin{array}{l}-[52],[67] \\
\text { nr [10] }\end{array}$ & & \\
\hline Public transport infrastructure $(n=2)$ & $-[11]$ & & $\mathrm{nr}[10]$ & & \\
\hline Parking difficult near local shopping areas $(n=1)$ & & $\mathrm{nr}[60]$ & $\mathrm{nr}[60]$ & & \\
\hline \multicolumn{6}{|l|}{ Socio-demographic environment } \\
\hline Neighbourhood SES $(n=7)$ & $\begin{array}{l}-[8]^{\mathrm{WaO}},-[34]^{\mathrm{WO}} \\
\operatorname{nr}[9]^{0},[59]\end{array}$ & & $\begin{array}{l}+[79]^{\mathrm{a}} \\
+\mathrm{u}[49]^{\mathrm{aO}} \\
-\mathrm{u}[49]^{\mathrm{aO}} \\
\operatorname{nr}[59]^{\mathrm{O}}\end{array}$ & $\begin{array}{l}+[59]^{\circ} \\
\operatorname{nr}[49]^{\mathrm{aO}}\end{array}$ & $\begin{array}{l}+[59]^{\circ} \\
\operatorname{nr}[82]^{\circ}\end{array}$ \\
\hline Neighbourhood deprivation $(n=3)$ & $+[80]^{\mathrm{aO}},[22]^{\mathrm{aO}}$ & & $\begin{array}{l}+[18]^{(\mathrm{med})} \\
\mathrm{nr}[22]^{\mathrm{aO}} \text { occ }\end{array}$ & & $\operatorname{nr}[22]^{\mathrm{aO}}$ \\
\hline Residential density $(n=3)$ & $\operatorname{nr}[11]$ & $\begin{array}{l}-[83]^{0} \\
\operatorname{nr}[60]^{a}\end{array}$ & $+[60]^{a}$ & & \\
\hline \multicolumn{6}{|l|}{ Neighbourhood safety } \\
\hline Safe park $(n=1)$ & $-[11]$ & & & & \\
\hline Neighbourhood safety $(n=8)$ & $\operatorname{nr}[9],[11],[62]^{\text {Wa }}$ & $\begin{array}{l}+[85]^{\mathrm{W}} \\
\mathrm{nr}[60]^{\mathrm{a}}\end{array}$ & $\begin{array}{l}-[60]^{\text {Wa }} \\
\text { nr [10], [47] }\end{array}$ & $\operatorname{nr}[16]$ & \\
\hline Neighbourhood problems $(n=1)$ & $+[84]^{\mathrm{w}}$ & & & & \\
\hline
\end{tabular}

Note: Each result is reported as positive (+), negative (-), or not related (nr) for objective or self-reported/perceived environmental measure. Objective measures are identified as $\left(^{\circ}\right)$. Significant associations only in subgroup are identified as men $(\mathrm{M})$, women $(\mathrm{w})$, non-workers (nwr), and other (u). ${ }^{\circ c c}$ refers to occupational time. S refers to significant differences between groups. For two studies [18, 62] the studied factor was investigated as a mediator and identified as (med). ${ }^{a}$ Composite environmental measure (e.g., neighbourhood deprivation index), ${ }^{b}$ Feature included in a composite environmental measure 
One of the five studies reported an unexpected positive association [82]: the higher the neighbourhood walkability index, the higher the time spent sitting and finally, two studies reported no associations with walkability and total screen time [8] or total sedentary time assessed objectively [59]. Only one of four studies considering walking and cycling facilities found a negative association between bike facilities and sedentary behaviour [11] and no significant associations were found with footpaths. Only one study explored the interaction between work status and neighbourhood walkability reporting a significant correlation [9]. Air or noise pollution and weather were found to be significantly associated with an increase in sedentary time. Findings relating to traffic safety were contradictory $[10,48,60]$. Lastly, no clear patterns of association were observed regarding street connectivity or land mix and sedentary behaviours.

\section{Services available in the environment}

Regarding the services/destinations resources, only Van Dyck et al. [60] found interactions with gender resulting in significant negative linear relations among men between access, proximity and number of destination close to home and sitting time; whereas six studies found no association with sedentary behaviours. Fields et al. [11] reported a negative association between public transport facilities (i.e., bus stop) and leisure time spent sedentary whereas Wallmann-Sperlich et al. [10] found no significant association with sitting time and public transport facilities.

\section{Socio-demographic environment}

Neighbourhood socioeconomic status was one of the most commonly investigated environmental factor $(n=7$ studies) and results are contradictory. Of the seven studies, two studies reported a negative association between neighbourhood socioeconomic status and TV viewing among women [8, 34]. Proper et al. [49] reported greater sitting time for disadvantaged neighbourhoods during weekend days and less sitting time during weekdays while Kozo et al. [59] and Stamatakis et al. [79] found positive associations irrespective of the day of the week. Three studies also reported a positive association between neighbourhood deprivation and leisure screen time and total sitting time $[18,22,79,80]$. One study reported a significant positive association between residential density and total sitting time [60] whereas results were mixed for time spent sedentary in transport [60, 83] and null for leisure screen time [11].

\section{Neighbourhood safety}

Of eight studies considering neighbourhood/park safety, patterns of associations showed mixed results depending on the type of sedentary behaviour being measured. Fields et al. [11] and van Dyck et al. [60] reported that leisure screen time and total siting time respectively were negatively associated with neighbourhood safety whereas Strong [84] reported a positive correlation for women between neighbourhood problems such as crime and television viewing time. In the opposite, Lee et al. [85] reported a positive association between neighbourhood safety and time spent in car among woman. Seven studies found no association between neighbourhood safety and sedentary behaviours.

\section{Home/work indoor environment}

Two studies found associations between indoor equipment and sedentary behaviours. Van Dyck et al. [55, 82] found that after adjustment for socio-demographic factors, the size of the largest TV set and the number of computers in the home was positively associated with TV viewing time and leisure-time internet usage. Regarding the indoor work-environment, Crespo et al. [86] found a worksite promotion index including shower facilities at work, lockers for clothes at work and safe bicycle storage to be associated with greater sedentary time, but at the same time also associated with increased levels of recreational physical activity. Finally Saidj et al. found a negative association between habitat surface area and leisure time sitting cross-sectional, while the association was no more significant in a longitudinal perspective [17].

\section{Discussion}

The aim of this review was to summarise the available literature on factors associated with sedentary behaviour in adults aged between 18 and 65 years. We aimed to provide updated information on previously reported factors and identify new ones that should be considered in the development of novel anti-sedentary behaviour interventions for this population. All published literature that met our inclusion criteria was included and themed based on a socio-ecological framework taking into account the different levels of correlates (intrapersonal, interpersonal, environmental and policy) as described by Owens and colleagues [1]. Seventy-four peer reviewed papers focusing on factors that influence sedentary behaviours in healthy adults were analysed, the majority of which were cross- sectional in design, with a significant increase in prospective analyses in the last 2 years. Studies were all conducted across four continents (Australia, Asia, Europe and North America), the majority of which were high-income countries, highlighting the call for studies investigating sedentary behaviours in low or middle-income countries.

Existing literature reports individual correlates such as age, body mass index, physical activity levels, mood and 
attitude were the most frequently investigated correlates $[2,6]$. Rhodes [6] reported that research was very limited in terms of interpersonal and environmental factors that may influence sedentariness in this population [6]. We however have found that recent efforts have focused on these types of correlates specifically environmental factors and there has been an increase in the number of published papers in the past three years reporting these factors and their associations with sedentary behaviours in adults [8-16, 42, 59, 60, 63, 77, 79, 83-85].

In terms of intrapersonal or individual factors, findings were similar to those previously reported demonstrating that those who were more sedentary in terms of either total time or leisure time sedentariness were older, female, did not participate in physical activity or exercise on a regular basis, had a higher body mass index, tended to smoke, consume high calorie snacks and use their cell phone more regularly. Men and especially those with a higher income were more prone to spending time sitting as a means of transport $[15,59,60,77]$.

Very few studies examined psychological factors associated with sedentary behaviour and analyses of those that did were difficult to compare as they all defined the psychological factors being investigated very differently [31, 42, 50, 57, 62]. For example, perceived depression was measured in four studies using four different outcomes measures (two used valid reliable measures while the other asked one question related to feelings of depression and anxiety). The heterogeneity of the factors, population samples and measurement tools used for both the factor and sedentary behaviour makes interpretation and conclusion problematic highlighting the importance of further research into these factors.

The relationship between socioeconomic status (as measured by income, occupation and education) and sedentary behaviour was entirely related to the domain of sedentary time measured; TVSE and educational levels had an inverse relationship whereas self-reported or objectively measured total sedentary time $[9,22-24$, $31,48,79]$ was positively correlated. The reason for this discrepancy appears that the higher the education level the more likely one is employed in professional more sedentary roles and this occupational sitting would be included in the measurement of total sedentary time. This emphasises the importance of studies focusing clearly on separate domains of sedentariness. Overall studies showed that socioeconomic status is a significant indicator, perhaps the most consistent factor of all the individual factors identified $[9,23,31,48,60]$.

Examination of interpersonal factors revealed consistencies throughout. Family related factors were the most frequently investigated, most specifically marital status, cohabiting and number of children in a household. Results were inconsistent highlighting the potential interaction with other individual or environmental factors that may influence the relationship. The influence of other interpersonal factors such as social norms [16, $52,55]$ and interaction with friends, peers and colleagues $[9,16,62,71,84]$ showed no overall relationship to sedentary behaviour. This is an unexpected finding as these factors would be presumed to have a considerable influence on sedentary behaviour as they have been shown to correlate closely with other unhealthy behaviours such as physical activity [87]. Further research into interpersonal factors is required, specifically their interaction with individual and environmental variables.

Of the environmental correlates, the socioeconomic domain was the most common domain studied. Some authors reported a low SES neighbourhood to be associated with an increase in TV viewing time $[8,34,80]$ while others found that a high SES neighbourhood resulted in increased sedentary behaviours [49, 59, 79]. More consistently, neighbourhood deprivation was positively associated with leisure screen time [22, 79, 80].

Multiple environmental attributes such as highly walkability neighbourhoods $[9,34,59,82]$, presence of aesthetic features $[9,10,16,60,62,85]$, proximity/access to destination and facilities [10, 11, 16, 47, 60,62], traffic safety $[9-11,60]$, residential density $[11,60,83]$ and a safe environment [9-11, 16, 47, 60,62, 85] presented numerous inconsistencies in their association with sedentary behaviours, ranging from significant to nonsignificant to contradictory findings. Multiple environmental factors (i.e., type of residence [10], overall indoor environment $[17,55,86]$, type of residence [10], presence of barriers/cul-de-sac [60], street connectivity $[60,83]$, presence of parking facilities [60], presence of public transportation infrastructure $[10,11]$ land use mix [83], air/noise pollution [47], and season [13], neighbourhood problems [84] were examined in only one or two studies each, preventing any conclusions being drawn on the significance, the direction or the strength of the association. Thus, further research is required to determine their potential impact on sedentary behaviour. More consistently, presence/proximity of green spaces $[12,14]$ was repeatedly negatively associated with sedentariness, and living in a rural area was consistently associated with an increase in sitting time in transport $[15,77]$. Finally weather was recurrently reported as a barrier and positively associated with total sitting time $[47,52,67,88]$.

The vast majority of studies that used objective environmental measures relied on administrative units or ego-centred definitions of the exposure area (i.e., street network or circular buffers centred on the participants' activity location) to better evaluate contextual effects on health [89]. Except from one study that examined the indoor worksite environment [86], all studies exclusively assessed the influence of residential neighbourhood on 
sedentary behaviour and did not account for other geographic life environments. Because individuals are mobile and thus exposed to various non-residential environments during their day-to-day activities [90] accounting solely for the residential environment could misrepresent the relationship between context and sedentary behaviours [91]. Finally, only one study investigated the impact of policy on sedentary behaviour [86]. Interestingly Crespo and colleagues [86] observed that a worksite programme promoting healthy living induced an increase in physical activity levels but at the same time resulted in a parallel increase in sedentary behaviour. Although there is only one study, this may be an important observation in that sedentary behaviour has been recognised as an independent risk factor for disease and should maybe tackled independently from physical inactivity and increasing physical activity may have an adverse effect on sedentariness.

Overall, there seems to be some preliminary evidence for the assertion that environmental characteristics related to both design and recreational resources may explain some of the variance in sedentary behavior. However the variance they explain above and beyond individual and intrapersonal factors remains difficult to ascertain and it is still unclear as to which changes in environments have the ability to affect sedentariness on a relatively permanent basis. Further research is needed to refine hypotheses about how specific environmental variables interact with individual and social factors and how they might be related to particular types and purposes of sedentary behaviour. In addition, policy makers need to begin to consider how to build communities so they facilitate transportation, occupation and recreation options that decrease prolonged sitting time.

With regard to interaction, limited research has been conducted investigating individual, interpersonal and environmental link factors. Kozo et al. [59] identified a relationship between neighbourhood walkability and household income and with each of these two factors independently with sedentary time. Similarly van Dyck et al. [60] reported several associations between different environmental factors, socio-demographic factors and socioeconomic status. Level of educational attainment was found to be inversely associated with income and employment and in turn all three variables were found to be closely related to TVSE by Stamatakis et al. [79] and several other authors. Gender was also shown to be a significant correlate with other intrapersonal factors such as BMI, physical activity levels and lifestyle choices such as smoking and high calorie snacking. Only two studies explored the potential interaction between individual, interpersonal and environmental correlates and age, education, occupation status, working status, retirement position and sedentary behaviours, and found no significant associations [16, 17]. Further analysis is warranted to establish the extent of the influence interactions between the various factors from the different socio-ecological levels have on sedentary behaviour.

Of interest is the finding that there was overall good consistency between self-report measure and objective measures (accelerometry) for most factors but not for all. For example Kozo et al. [59] reported an inverse relationship between neighbourhood walkability and selfreport sedentary time but the opposite was true for the relationship between neighbourhood walkability and objectively measured sedentariness. In addition, it is now well acknowledged that the context of the sedentary behaviour is critical to better understand the impact specific factors have on it. As is obvious from the variation of definitions across studies, the notion of sedentariness is plural and refers to different types of sedentary behaviours and correlates [92]. Moreover, though the science around sedentary behaviour is rapidly evolving, some sort of activities (i.e., screen time) that are currently under the sedentary behaviour banner should be reconsidered based on whether they are actually performed in sitting, lying or standing. In order to devise successful interventions to address sedentariness, more detailed and standardised contextual information is essential. In line with Chastin et al. [92], distinguishing between sedentary behaviours by purpose (i.e., work, leisure), environment (i.e., location, type of community, physical environment), type (i.e., screen based or not) and time (i.e., time of the day) would allow researchers to further identify the determinants and correlates of a specific sedentary behaviour and mitigate inconsistencies from previous studies.

The strengths of the review include the large number of abstracts and articles that were screened, the original approach that accounts for levels of correlates based on the socio-ecological model and the discovery of numerous environmental correlates that may need to be considered alongside individual and interpersonal factors when considering interventions to influences sedentary behaviours. Moreover, since the socio-ecological model describes the interrelations between the intra-personal, inter-personal, environmental and policy correlates of health behaviours [1], we also specifically aimed to explore links and interactions between the different levels of correlates.

Several limitations regarding the publication bias and the sampling strategy are acknowledged. Firstly, the paper selection is based on the search terms in the method section which, if absent from the title or the abstract were not detectable and thus relevant studies may have been missed. Secondly, the review is limited to published work, potentially leading to an over representation of significant results and publication bias. Several 
studies not examining sedentary behaviours as a first outcome and reporting only preliminary analyses are included but where possible, results for adjusted models are reported. In terms of available evidence, the vast majority of studies relied on cross sectional design that limits causal inference and is subject to reverse causality. Consequently the current evidence base is merely about factors associated or correlated with sedentariness rather than determinants or causes of change of sedentary behaviour over time. Finally the heterogeneity of the measurement tools and the plurality of sedentary behaviour definitions make cross study comparison and analyses difficult.

\section{Conclusions}

Despite the fact that numerous questions remain about what determines sedentary behaviour, results from this review provide a plethora of information relating to the multiple factors associates with sedentariness. This information base has increased significantly over the past 5 years since the last published review. It is clear that numerous correlates from individual level through to environmental level factors are important.

More focused research in the future will need to identify the specific settings and type of sedentary behaviour and explore correlates and determinants of settingspecific behaviours.

Longitudinal study designs will allow researchers to identify true determinants and clearly separate them from correlates. Homogeneity in terms of outcome measures would allow more in depth analysis including reporting of effect size and providing more meaningful and useful conclusions. The current evidence base is sufficient to include individual, intrapersonal and environmental factors in the equation when developing and testing interventional designs. However further analysis of interaction between these multiple level factors is essential to optimising any programme or policy focused on disrupting sedentary behaviours and in turn improving population health.

\section{Additional file}

Additional file 1: Table S1. Systematic search terms. Table S2. Data Extraction headings. Table S3. Checklist for assessing the quality of QUANTITATIVE studies. Table S4. Checklist for assessing the quality of QUALITATIVE studies. (PDF $305 \mathrm{~kb}$ )

\section{Competing interest}

The authors declare that they have no competing interests. We, the authors have received no reimbursements, fees, funding or salary from any organisation that may gain financially for the publication of this manuscript. We do not hold any stocks or shares in an organisation that may in any way gain or lose financially from the publication of this manuscript and finally, we are not applying for any patents relating to the content of the manuscript.

\section{Authors' contributions}

The manuscript was written by GO'D, JAN and CP under the editorial steer of DO'G, CS and SC. GO'D wrote the protocol and conducted the initial database searches. GO'D and JAN performed all data extraction in collaboration with KM and CP while $J$, HvdP and CB performed the role of secondary assessors. All authors read and approved the final manuscript.

\section{Acknowledgements}

"The preparation of this paper was supported by the DEterminants of Dlet and Physical ACtivity (DEDIPAC) knowledge hub. This work is supported by the Joint Programming Initiative 'Healthy Diet for a Healthy Life'. The funding agencies supporting this work are (on alphabetical order of participating Member State): France: Institut National de la Recherche Agronomique (INRA), Institut National de Prevention et d'Education pour la Sante (INPES); Ireland: The Health Research Board (HRB); The Netherlands: The Netherlands Organisation for Health Research and Development (ZonMw); The United Kingdom: The Medical Research Council (MRC)." Additional support has been provided by DEXLIFE (EU FP7 Programme) Grant agreement no: 27922.

\section{Author details}

${ }^{1}$ Centre for Preventive Medicine, School of Health \& Human Performance, Dublin City University, Dublin 9, Republic of Ireland. ${ }^{2}$ CarMeN Laboratory, INSERM U1060, Lyon 1 University, CRNH-Rhône-Alpes, CENS, Lyon, France. ${ }^{3}$ EMGO Institute for Health and Care Research, VU University Medical Centre, Amsterdam, Netherlands. ${ }^{4}$ TNO, Leiden, The Netherlands. Institute of Applied Health Research, School of Health and Life Science, Glasgow Caledonian University, Glasgow, UK.

Received: 29 July 2015 Accepted: 5 February 2016

Published online: 17 February 2016

\section{References}

1. Owen N, Sugiyama T, Eakin EE, Gardiner PA, Tremblay MS, Sallis JF. Adults' sedentary behavior: determinants and interventions. Am J Prev Med. 2011:41(2):189-96.

2. Thorp AA, Owen N, Neuhaus M, Dunstan DW. Sedentary behaviors and subsequent health outcomes in adults: a systematic review of longitudinal studies, 19962011. Am J Prev Med. 2011;41(2):207-15.

3. Wilmot EG, Edwardson CL, Achana FA, Davies MJ, Gorely T, Gray LJ, et al. Sedentary time in adults and the association with diabetes, cardiovascular disease and death: Systematic review and meta-analysis. Diabetologia. 2012;55(11):2895-905

4. Network SBR. Standardized use of the terms "sedentary" and "sedentary behaviours". Appl Physiol Nutr Metab. 2012:37:540-2.

5. Pate RR, O'Neill JR, Lobelo F. The evolving definition of "sedentary". Exerc Sport Sci Rev. 2008;36(4):173-8.

6. Rhodes RE, Mark RS, Temmel CP. Adult sedentary behavior a systematic review. Am J Prev Med. 2012:42(3):E3-28.

7. Glass TA, McAtee MJ. Behavioral science at the crossroads in public health: extending horizons, envisioning the future. Soc Sci Med. 2006:62:1650-71.

8. Coogan PF, White LF, Evans SR, Palmer JR, Rosenberg L. The influence of neighborhood socioeconomic status and walkability on TV viewing time. J Phys Act Health. 2012;9(8):1074-9.

9. Ding D, Sugiyama T, Winkler E, Cerin E, Wijndaele K, Owen N. Correlates of change in adults' television viewing time: a four-year follow-up study. Med Sci Sports Exerc. 2012;44(7):1287-92.

10. Wallmann-Sperlich B, Bucksch J, Hansen S, Schantz P, Froboese I. Sitting time in Germany: an analysis of socio-demographic and environmental correlates. BMC Public Health. 2013;13:196.

11. Fields R, Kaczynski AT, Bopp M, Fallon E. Built environment associations with health behaviors among Hispanics. J Phys Act Health. 2013;10(3):335-42.

12. Storgaard RL, Hansen HS, Aadahl M, Glumer C. Association between neighbourhood green space and sedentary leisure time in a Danish population. Scand J Public Health. 2013:41(8):846-52.

13. Hagströmer M, Rizzo NS, Sjöström M. Associations of season and region on objectively assessed physical activity and sedentary behaviour. J Sports Sci. 2014;32(7):629-34

14. Astell-Burt T, Feng X, Kolt GS. Greener neighborhoods, slimmer people evidence from 246920 Australians. Int J Obes. 2014;38(1):156-9.

15. Zolnk EJ. The effects of sprawl on private vehicle commuting distances and times. Plan Des. 2011;38:1071. 
16. Van Holle V, McNaughton SA, Teychenne M, Timperio A, Van Dyck D, De Bourdeaudhuij I, et al. Social and physical environmental correlates of adults' weekend sitting time and moderating effects of retirement status and physical health. Int J Environ Res Public Health. 2014;11(9):9790-810.

17. Saidj M, Jørgensen $T$, Jacobsen RK, Linneberg A, Aadahl M. The influence of housing characteristics on leisure-time sitting. A prospective cohort study in Danish adults. Prev Med. 2015;81:58-62.

18. Oliver MWK, Blakely T, Parker K, Badland H, Schofield G, Ivory V, et al. Neighbourhood built environment associations with body size in adults: mediating effects of activity and sedentariness in a cross-sectional study of New Zealand adults. BMC Public Health. 2015;15(1):1-11 11.

19. Lakerveld J, Ploeg HPV, consortium obotD. Towards the integration and development of a cross-European research network and infrastructure: the DEterminants of Dlet and Physical ACtivity (DEDIPAC) Knowledge Hub. Int J Behav Nutr Phys Act. 2014;11:143-53.

20. Kmet L, Lee RC, Cook LS. Standard quality assessment criteria for evaluating primary research papers from a variety of fields. Institute of Health Economics 2004;1-31.

21. Lakshman R, Paes VM, Hesketh K, O'Malley C, Moore H, Ong K, et al. Protocol for systematic reviews of determinants/ correlates of obesityrelated dietary and physical activity behaviors in young children (preschool 0 to 6 years): evidence mapping and syntheses. Syst Rev. 2013;2:28-35.

22. Stamatakis E, Coombs N, Rowlands A, Shelton N, Hillsdon M. Objectivelyassessed and self-reported sedentary time in relation to multiple socioeconomic status indicators among adults in England: a cross-sectional study. BMJ Open. 2014;4(11):e006034.

23. Xie YJ, Stewart SM, Lam TH, Viswanath $\mathrm{K}$, Chan SS. Television viewing time in Hong Kong adult population: Associations with body mass index and obesity. PLoS ONE. 2014;9(1), e85440. doi:10.1371/journal.pone.0085440.

24. Van Uffelen JGZ, Heesch KC, Brown W. Correlates of sitting time in working age australian women: Who should be targeted with interventions to decrease sitting time? J Phys Act Health. 2012;9(2):270-87.

25. Seguin R, Buchner DM, Liu J, Allison M, Manini T, Wang CY, et al. Sedentary behavior and mortality in older women: The women's health initiative. Am J Prev Med. 2014;46(2):122-35.

26. Ding D, Gebel K, Phongsavan P, Bauman AE, Merom D. Driving: A road to unhealthy lifestyles and poor health outcomes. PLoS ONE. 2014;9(6), e94602. doi:10.1371/journal.pone.0094602.

27. Uijtdewilligen L, Twisk JWR, Singh AS, Chinapaw MJM, van Mechelen W, Brown WJ. Biological, socio-demographic, work and lifestyle determinants of sitting in young adult women: A prospective cohort study. Int J Behav Nutr Phys Act. 2014. doi:10.1186/1479-5868-11-7.

28. Pomerleau J, McKee M, Robertson A, Vaasc S, Kadziauskiene K, Abaravicius A, et al. Physical inactivity in the Baltic countries. Prev Med. 2000;31 (6):665-72.

29. Hamer M, Stamatakis E, Mishra GD. Television- and screen-based activity and mental well-being in adults. Am J Prev Med. 2010;38(4):375-80.

30. Grothe KB, Roach J, Low A, Himes S, Craft JM, Norman GJ, et al. Sedentary behavior and food cravings in diverse overweight women: a pilot study. Women Health. 2013;53(4):405-18.

31. Granner M, Mburia-Mwalili A. Correlates of television viewing among African american and Caucasian women. Women Health. 2010;50(8):783-94.

32. Bowman S. Television-viewing characteristics of adults: correlations to eating practices and overweight and health status. Prev Chronic Dis. 2006;3(2):1-11.

33. Kaufman A, Augustson EM, Patrick H. Unraveling the relationship between smoking and weight: the role of sedentary behavior. J Obes. 2012;2012.

34. Sugiyama T, Salmon J, Dunstan DW, Bauman AE, Owen N. Neighborhood walkability and TV viewing time among Australian adults. Am J Prev Med. 2007;33(6):444-9.

35. Ballard M, Gray M, Reilly J, Noggle M. Correlates of video game screen time among males: body mass, physical activity, and other media use. Eat Behav. 2009;10:161-7

36. Vandelanotte C, Sugiyama T, Gardiner P, Owen N. Associations of leisuretime internet and computer use with overweight and obesity, physical activity and sedentary behaviors: Cross-sectional study. J Med Internet Res. 2009;11(3):e28. doi:10.2196/jmir.1084.

37. Wilson HJ, Leonard WR, Tarskaia LA, Klimova TM, Krivoshapkin VG, Snodgrass JJ. Objectively measured physical activity and sedentary behaviour of Yakut (Sakha) adults. Ann Hum Biol. 2014;41(2):178-84.

38. Chau JY, van der Ploeg HP, Merom D, Chey T, Bauman AE. Cross-sectional associations between occupational and leisure-time sitting, physical activity and obesity in working adults. Prev Med. 2012;54(3-4):195-200.
39. Clemes SA, O'Connell SE, Edwardson CL. Office Workers' objectively measured sedentary behavior and physical activity during and outside working hours. J Occup Environ Med. 2014;56(3):298-303.

40. Hadgraft NT LB, Clark BK, Healy GN, Owen N, Dunstan DW. Excessive sitting at work and at home: Correlates of occupational sitting and TV viewing time in working adults. BMC Public Health. 2015;15(1):1-13 13.

41. Menai M, Fezeu L, Charreire H, Kesse-Guyot E, Touvier M, Simon C, et al. Changes in sedentary behaviours and associations with physical activity through retirement: a 6-year longitudinal study. Plos One. 2014;9(9):e106850.

42. Li K, Davison KK, Jurkowski JM. Mental health and family functioning as correlates of a sedentary lifestyle among Low-income women with young children. Women Health. 2012;52(6):606-19.

43. Hirooka N, Takedai T, D'Amico F. Assessing physical activity in daily life, exercise, and sedentary behavior among Japanese moving to westernized environment: a cross-sectional study of Japanese migrants at an urban primary care center in Pittsburgh. Asia Pac Fam Med. 2014;13(1):3-3.

44. Kozey-Keadle S, Staudenmayer J, Libertine A, Mavilia M, Lyden K, Braun B, et al. Changes in sedentary time and physical activity in response to an exercise training and/or lifestyle intervention. J Phys Act Health. 2013.

45. Conroy DE, Maher JP, Elavsky S, Hyde AL, Doerksen SE. Sedentary behavior as a daily process regulated by habits and intentions. Health Psychol. 2013;32(11):1149-57.

46. Lepp A, Barkley JE, Sanders GJ, Rebold M, Gates P. The relationship between cell phone use, physical and sedentary activity, and cardiorespiratory fitness in a sample of U.S. college students. Int J Behav Nutr Phys Act. 2013. doi:10.1186/1479-5868-10-79.

47. Salmon J, Crawford D, Owen N, Bauman A, Sallis JF. Physical activity and sedentary behavior: a population-based study of barriers, enjoyment, and preference. Health Psychol. 2003;22(2):178-88.

48. Clark BK, Sugiyama T, Healy GN, Salmon J, Dunstan DW, Shaw JE, et al. Sociodemographic correlates of prolonged television viewing time in australian men and women: The AusDiab study. J Phys Act Health. 2010;7(5):595-601.

49. Proper K, Cerin E, Brown W, Owen N. Sitting time and socio-economic differences in overweight and obesity. Int J Obes. 2007;31:169-76.

50. Hamrik Z, Sigmundová D, Kalman M, Pavelka J, Sigmund E. Physical activity and sedentary behaviour in Czech adults: Results from the GPAQ study. Euro J Sport Sci. 2014;14(2):193-8.

51. Kouvonen A, Kivimäki M, Virtanen M, Heponiemi T, Elovainio M, Pentti J, et al. Effort-reward imbalance at work and the co-occurrence of lifestyle risk factors: cross-sectional survey in a sample of 36,127 public sector employees. BMC Public Health. 2006;6:24.

52. Mabry RM, Al-Busaidi ZQ, Reeves MM, Owen N, Eakin EG. Addressing physical inactivity in Omani adults: perceptions of public health managers. Public Health Nutr. 2014;17(3):674-81.

53. Saidj MMM, Charreire H, Weber C, Enaux C, Aadahl M, Kesse-Guyot E, et al. Descriptive study of sedentary behaviours in 35,444 French working adults: crosssectional findings from the ACTI-Cités study. BMC Public Health. 2015;15(1):1-10.

54. De Cocker K, Duncan MJ, Short C, van Uffelen JGZ, Vandelanotte C. Understanding occupational sitting: Prevalence, correlates and moderating effects in Australian employees. Prev Med. 2014;67:288-94.

55. Van Dyck D, Cardon G, Deforche B, Owen N, De Cocker K, Wijndaele K, et al. Socio-demographic, psychosocial and home-environmental attributes associated with adults' domestic screen time. BMC Public Health. 2011;11:668.

56. Ekelund U, Brage S, Besson H, Sharp S, Wareham NJ. Time spent being sedentary and weight gain in healthy adults: Reverse or bidirectional causality? Am J Clin Nutr. 2008:88(3):612-7.

57. de Wit L, van Straten A, Lamers F, Cuijpers P, Penninx B. Are sedentary television watching and computer use behaviors associated with anxiety and depressive disorders? Psychiatry Res. 2011;186(2-3):239-43.

58. Zolnk EJ. The effects of sprawl on private vehicle commuting distances and times. Plan Des. 2011;38:1071.

59. Kozo J, Kerr J, Saelens BE, Sallis JF, Conway TL, Cain K, et al. Sedentary behaviors of adults in relation to neighborhood walkability and income. Health Psychol. 2012;31(6):704-13.

60. Van Dyck D, Cerin E, Conway TL, De Bourdeaudhuij I, Owen N, Kerr J, et al. Associations between perceived neighborhood environmental attributes and adults' sedentary behavior: Findings from the USA, Australia and Belgium. Soc Sci Med. 2012;74(9):1375-84.

61. Sugiyama T, Healy GN, Dunstan DW, Salmon J, Owen N. Is television viewing time a marker of a broader pattern of sedentary behavior? Ann Behav Med. 2008;35(2):245-50. 
62. Teychenne M, Ball K, Salmon J. Correlates of socio-economic inequalities in women's television viewing: A study of intrapersonal, social and environmental mediators. Int J Behav Nutr Phys Act. 2012;3.

63. Ishii K, Shibata A, Oka K. Sociodemographic and anthropometric factors associated with screen-based sedentary behavior among Japanese adults: A population-based cross-sectional study. J Epidemiol. 2013;23(5):382-8.

64. De Cocker KA, van Uffelen JGZ, Brown WJ. Associations between sitting time and weight in young adult Australian women. Prev Med. 2010;51(5): 361-7.

65. Sugiyama T, Merom D, Reeves M, Leslie E, Owen N. Habitual active transport moderates the association of TV viewing time with body mass index. J Phys Act Health. 2010;7(1):11-6.

66. Munir F, Houdmont J, Clemes S, Wilson K, Kerr R, Addley K. Work engagement and its association with occupational sitting time: results from the Stormont study. BMC Public Health. 2015:15:30.

67. George ES, Kolt GS, Rosenkranz RR, Guagliano JM. Physical activity and sedentary time: male perceptions in a university work environment. Am J Mens Health. 2014;8(2):148-58.

68. Clark BK, Peeters GMEE, Gomersall SR, Pavey TG, Brown WJ. Nine year changes in sitting time in young and mid-aged Australian women: Findings from the Australian Longitudinal Study for Women's Health. Prev Med. 2014; 64:1-7.

69. den Hoed M, Brage S, Jing Hua Z, Westgate $K$, Ay run N, Ekelund U, et al. Heritability of objectively assessed daily physical activity and sedentary behavior. Am J Clin Nutr. 2013;98(5):1317-25.

70. Evenson KR, Wen F. Prevalence and correlates of objectively measured physical activity and sedentary behavior among US pregnant women. Prev Med. 2011:53(1-2):39-43.

71. Uijtdewilligen L, Singh AS, Chinapaw MJ, Koppes LLJ, van Mechelen W, Twisk JWR. Number and appraisal of daily hassles and life events in young adulthood: the association with physical activity and screen time: a longitudinal cohort study. BMC Public Health. 2014;14:1067.

72. Rhodes RE, Dean RN. Understanding physical inactivity: Prediction of four sedentary leisure behaviors. Leis Sci. 2009;31(2):124-35.

73. Barnett I, van Sluijs E, Ogilvie D, Wareham NJ. Changes in household, transport and recreational physical activity and television viewing time across the transition to retirement: longitudinal evidence from the EPIC-Norfolk cohort. J Epidemiol Community Health. 2014.

74. Jans MP, Proper Kl, Hildebrandt VH. Sedentary behavior in Dutch workers. Differences between occupations and business sectors. Am J Prev Med. 2007:33(6):450-4.

75. Thorp AA, Healy GN, Winkler E, Clark BK, Gardiner PA, Owen N, et al. Prolonged sedentary time and physical activity in workplace and non-work contexts: A cross-sectional study of office, customer service and call centre employees. Int J Behav Nutr Phys Act. 2012;9.

76. Parry $S$, Straker $L$. The contribution of office work to sedentary behaviour associated risk. BMC Public Health. 2013;13.

77. Sugiyama TMD, van der Ploeg HP, Corpuz G, Bauman A, Owen N. Prolonged sitting in cars: prevalence, socio-demographic variations and trends. Prev Med. 2012;55:215.

78. Touvier M, Bertrais S, Charreire H, Vergnaud A, Hercberg S, Oppert J. Changes in leisure-time physical activity and sedentary behaviour at retirement: A prospective study in middle-aged French subjects. Int J Behav Nutr Phys Act. 2010;7.

79. Stamatakis E, Grunseit AC, Coombs N, Ding D, Chau JY, Phongsavan P, et al. Associations between socio-economic position and sedentary behaviour in a large population sample of Australian middle and older-aged adults: The Social, Economic, and Environmental Factor (SEEF) Study. Prev Med. 2014;63:72-80.

80. Stamatakis E, Hillsdon M, Mishra G, Hamer M, Marmot M. Television viewing and other screen-based entertainment in relation to multiple socioeconomic status indicators and area deprivation: The Scottish Health Survey 2003. J Epidemiol Community Health. 2009;63(9):734-40.

81. Leal C, Chaix B. The influence of geographic life environments on cardiometabolic risk factors: a systematic review, a methodological assessment and a research agenda. Obes Rev. 2011;12(3):217-30.

82. Van Dyck D, Cardon G, Deforche B, Owen N, Sallis JF, De Bourdeaudhuij I. Neighborhood walkability and sedentary time in Belgian adults. Am J Prev Med. 2010;39(1):25-32

83. Frank LDAM, Schmid TL. Obesity relationships with community design physical activity and time spent in cars. Am J Prev Med. 2004;27:749.
84. Strong LL RL, Wetter DW, McNeill LH. Associations of perceived neighbourhood physical and social environments with physical activity and television viewing in African American men and women. Am J Prev Med. 2013;27:1.

85. Lee REMS, Adamus-Leach HJ. Neighbourhood street scale elelments, sedentary time and cardiometabolic risk factors in inactive ethnic minority women. PLOS ONE. 2012;7, e51081.

86. Crespo NC, Sallis JF, Conway TL, Saelens BE, Frank LD. Worksite physical activity policies and environments in relation to employee physical activity. Am J Health Promot. 2011;25(4):264-71.

87. Trost SG, Owen N, Bauman AE, Sallis JF, Brown W. Correlates of adults participation in physical activity: review and update. Med Sci Sports Exerc. 2002;34:1996-2001.

88. George ES, Rosenkranz RR, Kolt GS. Male perceptions of physical activity and sedentary behavior in a university work environment. Med Sci Sports Exerc. 2013;45(5):48.

89. Chaix B. Geographic life environments and coronary heart disease: a literature review, theoretical contributions, methodological updates, and a research agenda. Annu Rev Public Health. 2009;30:81-105.

90. Perchoux C, Kestens Y, Thomas F, Hulst A, Thierry B, Chaix B. Assessing patterns of spatial behavior in health studies: Their socio-demographic determinants and associations with transportation modes (the RECORD Cohort Study). Soc Sci Med. 2014;119:64-73.

91. Perchoux C, Chaix B, Cummins S, Kestens Y. Conceptualization and measurement of environmental exposure in epidemiology: Accounting for activity space related to daily mobility. Health Place. 2013;21:86-93.

92. Chastin SFM, Schwarz U, Skelton DA. Development of a consensus taxonomy of sedentary behaviors (SIT): Report of Delphi round 1. PLOS ONE. 2013;8(12):e82313. doi:10.1371/journal.pone.0082313.

\section{Submit your next manuscript to BioMed Central and we will help you at every step:}

- We accept pre-submission inquiries

- Our selector tool helps you to find the most relevant journal

- We provide round the clock customer support

- Convenient online submission

- Thorough peer review

- Inclusion in PubMed and all major indexing services

- Maximum visibility for your research

Submit your manuscript at www.biomedcentral.com/submit 